\title{
Observational constraints on Cosmic
}

\section{Reionization}

\author{
Xiaohui Fan ${ }^{1}$, C.L. Carilli ${ }^{2}$, B. Keating ${ }^{3}$ \\ ${ }^{1}$ Steward Observatory, University of Arizona, Tucson, AZ 85721; \\ ${ }^{2}$ National Radio Astronomy Observatory, Socorro, NM, 87801; \\ ${ }^{3}$ Department of Physics, University of California, San Diego, CA, 92093
}

Key Words Cosmology - observational; Galaxy formation; cosmic reionization

\begin{abstract}
Recent observations have set the first constraints on the epoch of reionization (EoR), corresponding to the formation epoch of the first luminous objects. Studies of Gunn-Peterson (GP) absorption, and related phenomena, suggest a qualitative change in the state of the intergalactic medium (IGM) at $z \sim 6$, indicating a rapid increase in the neutral fraction of the IGM, from $x_{H I}<10^{-4}$ at $z \leq 5.5$, to $x_{H I}>10^{-3}$, perhaps up to 0.1 , at $z \geq 6$. Conversely, transmission spikes in the GP trough, and the evolution of the Ly $\alpha$ galaxy luminosity function indicate $x_{H I}<0.5$ at $z \sim 6.5$, while the large scale polarization of the cosmic microwave background (CMB) implies a significant ionization fraction extending to higher redshifts, $z \sim 11 \pm 3$. The results suggest that reionization is less an event than a process, with the process beginning as early as $z \sim 14$, and with the 'percolation', or 'overlap' phase ending at $z \sim 6$. The data are consistent with low luminosity star forming galaxies as being the dominant sources of reionizing photons. Low frequency radio telescopes currently under construction should be able to make the first direct measurements of $\mathrm{HI} 21 \mathrm{~cm}$ emission from the neutral IGM during the EoR, and
\end{abstract}


upcoming measurements of secondary CMB temperature anisotropy will provide fine details of the dynamics of the reionized IGM.

\section{CONTENTS}

Introduction

A basic model of reionization $\ldots \ldots \ldots \ldots \ldots$

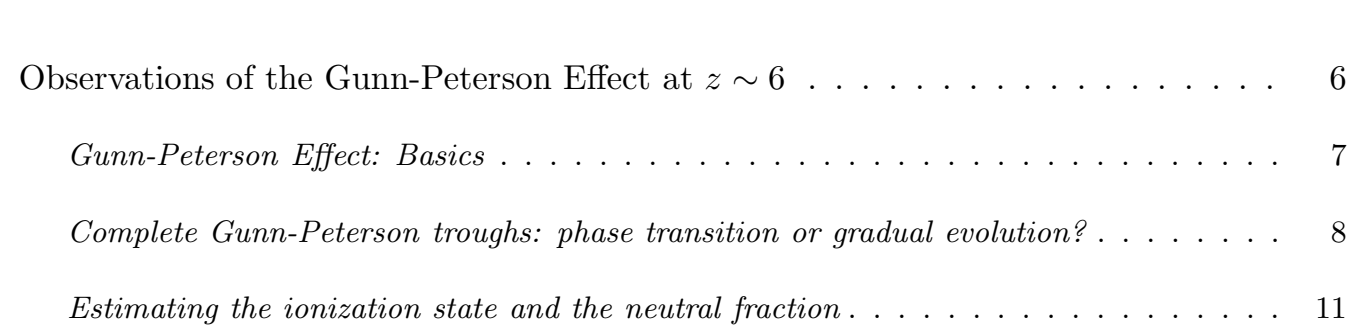

Cosmic Strömgren spheres and surfaces

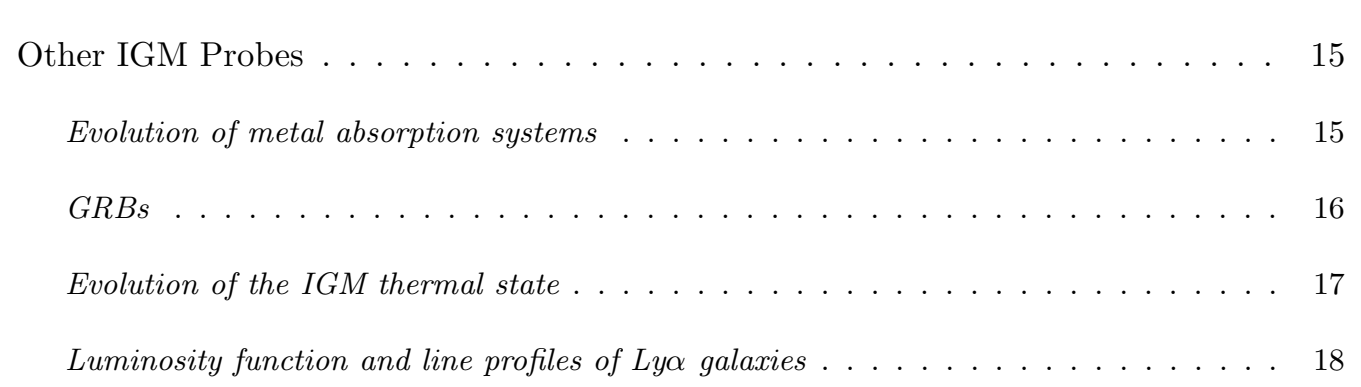

Cosmic Microwave Background Probes of Reionization . . . . . . . . . . . . . 20

The Generation of CMB Polarization and Temperature Anisotropy During the EoR . . 21

Current CMB Results and Future Prospects . . . . . . . . . . . . . . . . 28

Observational Challenaes . . . . . . . . . . . . . . . . . 30

Discussion and Future Prospects . . . . . . . . . . . . . . . . 31

Sources of Reionization . . . . . . . . . . . . . . . . . . . . . 34

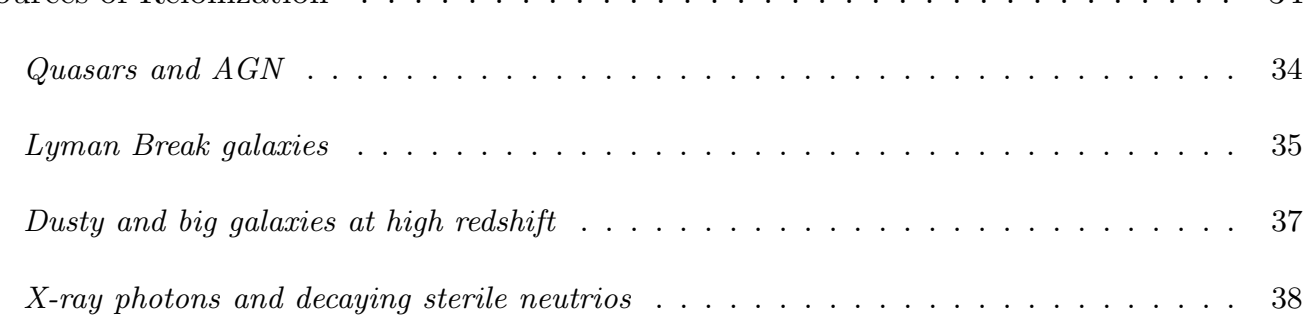




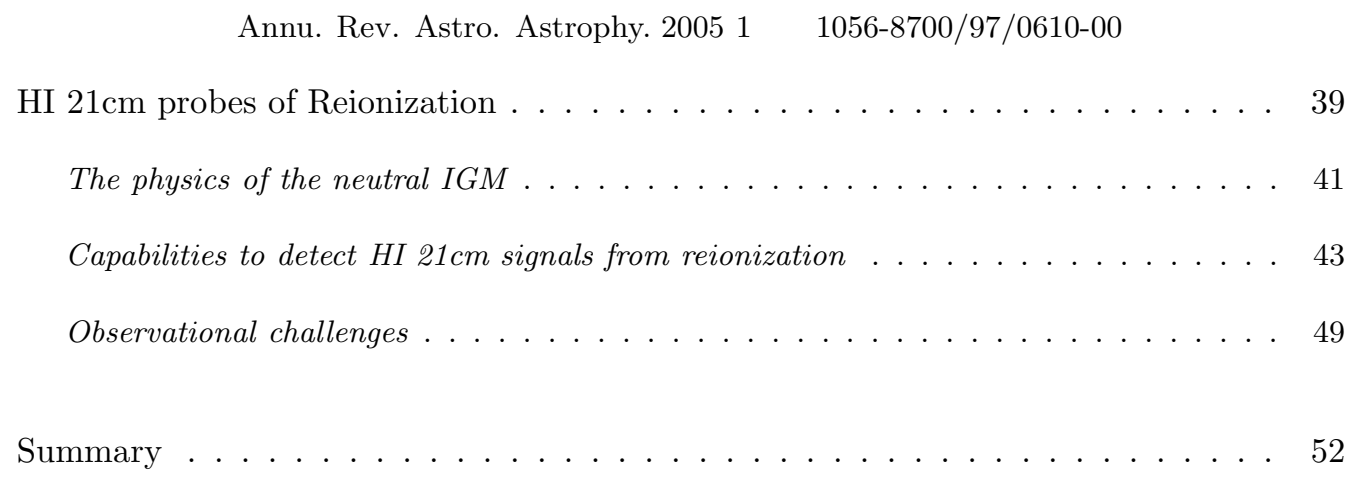

\section{Introduction}

The baryonic pre-galactic medium (PGM) evolves in three distinct phases. At high redshifts $(z>1100)$ the PGM is hot, fully ionized, and optically thick to Thomson scattering, and hence coupled to the photon field. As the universe expands, the PGM cools, and eventually recombines, leaving a surface of last scattering (the cosmic microwave background, CMB), plus a neutral PGM. This neutral phase lasts from $z=1100$ to $z \sim 14$. At some point between $z \sim 14$ and 6 , hydrogen in the PGM is 'reionized', due to UV radiation from the first luminous objects, leaving the fully reionized intergalactic medium (IGM) seen during the 'realm of the galaxies' $(6>z>0)$. The ionized, dense PGM at very high redshift has been well studied through extensive observations of the CMB. Likewise, the reionized, rarified IGM at low redshift has been well characterized through QSO absorption line studies. The middle phase - the existence of a neutral IGM during the so-called 'dark ages' (Rees 1998), and the process of reionization of this medium, is the last directly observable phase of cosmic evolution that remains to be verified and explored. The epoch of reionization (EoR) is crucial in cosmic structure formation studies, since it sets a fundamental benchmark indicating the formation of the first luminous objects, either star forming galaxies or active galactic nuclei (AGN). 
Cosmic reionization has been discussed within the larger context of mostly theoretical reviews on the formation of the first luminous objects (Loeb \& Barkana 2002; Barkana \& Loeb 2001; Ciardi \& Ferrara 2005). The past few years have seen the first observational evidence for a neutral IGM, and the first constraints on the process of reionization. In this review we do not repeat the theory of early structure formation, but focus on these recent observations of reionization and the evolution of the neutral IGM. Such constraints come from optical and near-IR spectroscopy of the most distant QSOs, and study of galaxy populations at the highest redshifts, among others. Detections of the IGM Thomson optical depth come from observations of the large angular scale polarization of the CMB (Kogut et al. 2003; Spergel et al. 2006; Page et al. 2006"). These measurements yield $\mathrm{a} \gtrsim 3 \sigma$ detection of polarized emission of the CMB, and future observations of the small scale CMB anisotropy will complement the large angular scale polarization by probing the fine-details of the reionization epoch. We also review the potential sources of reionization, including star forming galaxies, AGN, and decaying particles. We conclude with a discussion of low frequency radio telescopes that are being constructed to detect the neutral IGM directly through the $21 \mathrm{~cm}$ line of neutral hydrogen.

We focus our review on the observations of hydrogen reionization. Neutral helium reionization likely happened at a similar epoch to that of neutral hydrogen, due to their similar ionization potential and recombination rate. Reionization of He II happens at a much lower redshift - the He II Gunn-Peterson effect is observed in the spectrum of quasars at $z \sim 3$ (e.g. Jakobsen et al. 1994, Davidsen, Anderson et al. 1999, Kriss et al. 2001, Shull et al. 2004, Zheng et al. 2004). Evolution of the IGM temperture (e.g. Ricotti, Gnedin \& Shull 2000, Schaye et 
al. 2000, Theuns et al. 2002a,b) also suggest a rapid change of IGM temperture at $z \sim 3$, consistent with the onset of He II reionization. For a detailed recent review of HeII reionization, see Ciardi \& Ferrara (2005).

\section{A basic model of reionization}

In order to put recent observations in context, we include a brief, mostly qualitative, description of the reionization process. Again, for more extensive reviews of early structure formation, see Loeb \& Barkana (2002) Barkana \& Loeb( 2002), and Ciardi \& Ferrara (2005).

Assuming reionization is driven by UV photons from the first luminous sources (stars or AGN), analytic (eg. Madau, Haardt, \& Rees 1997; Miralda-Escude, Haenelt, \& Rees 2000; Wyithe \& Loeb 2003), and numerical (eg. Gnedin 2000; Razoumov et al. 2002; Sokasian et al. 2003; Ciardi, Stoehr, \& White 2004; Paschos \& Norman 2005), calculations suggest that reionization follows a number of phases. The first phase will be relatively slow, with each UV source isolated to its own Strömgren sphere. Eventually, these spheres grow and join, leading to much faster reionization, due to the combination of accelerating galaxy formation, plus the much larger mean free path of ionizing photons in the now porous IGM. This stage is known as 'overlap', or 'percolation'. The last phase entails the etching-away of the final dense filaments in the IGM by the intergalactic UV

radiation field, leading to a fully ionized IGM (neutral fraction, $x_{H I} n_{\mathrm{H}} \sim 10^{-5}$, at $z=0)$.

There are a number of important caveats to this most basic model. First, causality, cosmic variance, and source clustering will set an absolute minimum width in redshift for the fast phase of reionization, $\Delta z>0.15$, and the process 
itself is likely to be much more extended in time (Wyithe \& Loeb 2004). In particular, Furlanetto \& Oh (2005) point out that the overlap phase is a relatively local phenomenon, with significant differences along different lines of sight. Second, the recombination time for the mean IGM becomes longer than the Hubble time at $z \geq 8$, allowing for the possibility of complex reionization histories. As shown by a number of semi-analytical works (e.g., Cen 2003a,b, Chiu et al. 2003, Wyithe \& Loeb 2003, Haiman \& Holder 2003), adding feedback from an early generation of galaxy formation, or adjusting star formation efficiency at $z>6-10$, will produce a prolonged, or multi-epoch reionization history. Third, it remains an open question as to whether reionization proceeds from high to low density regions (inside-out; Ciardi \& Madau 2003, Sokasian et al. 2003; Iliev et al. 2006), or from low to high density regions (outside-in; Miralda-Escude et al. 2000, Gnedin 2000). Forth, a very different picture arises if we assume reionization by decaying fundamental particles, or by penetrating hard Xrays from the first luminous sources. In this case, the reionization process is not localized to individual Strömgren spheres, but occurs more uniformly throughout the IGM (§8.4). Lastly, we have been necessarily vague in terms of precise redshifts and timescales for the various phases - this is the main concern of the present review.

Throughout this review, we adopt a standard concordance cosmology (Spergel et al. 2003, Spergel et al. 2006), unless stated otherwise.

\section{Observations of the Gunn-Peterson Effect at $z \sim 6$}

Gunn \& Peterson (1965) first proposed using Lya resonance absorption in the spectrum of distant quasars as a direct probe to the neutral hydrogen density in the IGM at high-redshift (see also Field 1959, Shklovsky 1964, Scheuer 1965, 
Bahcall \& Salpeter 1965). For objects beyond reionization, neutral hydrogen in the IGM creates complete Gunn-Peterson absorption troughs in the quasar spectrum blueward of $\operatorname{Ly} \alpha$ emission. Observations of the Gunn-Peterson effect directly constrain the evolution of neutral hydrogen fraction and the ionization state of the IGM.

\subsection{Gunn-Peterson Effect: Basics}

The Gunn-Peterson (1965) optical depth to Ly $\alpha$ photons is

$$
\tau_{\mathrm{GP}}=\frac{\pi e^{2}}{m_{e} c} f_{\alpha} \lambda_{\alpha} H^{-1}(z) n_{\mathrm{HI}}
$$

where $f_{\alpha}$ is the oscillator strength of the Ly $\alpha$ transition, $\lambda_{\alpha}=1216 \AA, H(z)$ is the Hubble constant at redshift $z$, and $n_{\mathrm{HI}}$ is the density of neutral hydrogen in the IGM. At high redshifts:

$$
\tau_{\mathrm{GP}}(z)=4.9 \times 10^{5}\left(\frac{\Omega_{m} h^{2}}{0.13}\right)^{-1 / 2}\left(\frac{\Omega_{b} h^{2}}{0.02}\right)\left(\frac{1+z}{7}\right)^{3 / 2}\left(\frac{n_{\mathrm{HI}}}{n_{\mathrm{H}}}\right),
$$

for a uniform IGM. Even a tiny neutral fraction, $x_{H I} \sim 10^{-4}$, gives rise to complete Gunn-Peterson absorption. This test is only sensitive at the end of the reionization when the IGM is already mostly ionized, and saturates for the higher neutral fraction in the earlier stage.

At $z<5$, the IGM absorption is resolved into individual Ly $\alpha$ forest lines; their number density increases strongly with redshift $\left(N(z) \propto(1+z)^{2.5}\right.$, see Rauch 1998). Earlier attempts to study Gunn-Peterson absorption concentrated on measuring the amount of flux between individual Ly $\alpha$ forest lines using highresolution spectroscopy to place limits on the diffuse neutral IGM. Webb et al. (1992) and Giallongo et al. (1994) found $\tau<0.05$ at $z \sim 4$. Songaila et al. (1999) and Fan et al. (2000) place upper limits of $\tau=0.1$ and 0.4 based on lack 
of complete Gunn-Peterson troughs in SDSS J0338+0021 $(z=5.00)$ and SDSS J1044-0125 ( $z=5.74)$, respectively. However, at $z>5$, even with a moderately high resolution spectrum, Ly $\alpha$ forest lines overlap severely, making it impossible to find a truly "line-free" region.

A more accurate picture of the IGM evolution interprets the Ly $\alpha$ forest as a fluctuating Gunn-Peterson effect: absorption arises from low-density gas in the IGM that is in approximate thermal equilibrium between photoionization heating by the UV background and adiabatic cooling due to the Hubble expansion, rather than as discrete Ly $\alpha$ forest clouds (Bi 1993; Cen et al. 1994; Zhang et al. 1995; Herquist et al. 1996). The neutral hydrogen fraction and therefore the GP optical depth depends on the local density of the IGM. By studying the evolution of the average transmitted flux or effective optical depth, one can trace the evolution of the UV ionizing background and neutral fraction of the IGM. At high-redshift, the IGM is highly clumpy (Miralda-Escude et al. 2000), which has to be taken into account in order to estimate the IGM ionization from observations.

\subsection{Complete Gunn-Peterson troughs: phase transition or grad- ual evolution?}

The Sloan Digital Sky Survey (SDSS, York et al. 2000) provides large samples of luminous quasars over $0<z<6.5$. Fan et al. (2000, 2001, 2003, 2004, 2006a) carried out a survey of $i$-dropout quasars $(z>5.7)$ using the SDSS imaging data, resulting in the discovery of 19 luminous quasars in this redshift regime (Figure 11). Other multicolor survey projects (e.g, Mahabal et al. 2005; AGES, Cool et al. 2006; QUEST, Djorgovski et al. 2005; CFHT Legancy survey, Willott et al. 2005) are also searching for quasars at similar redshifts. They provide by far the 
best probes of IGM evolution towards the end of the EoR.

Songaila (2004) summarized the evolution of transmitted flux over a wide redshift range $(2<z<6.3)$ using high signal-to-noise, moderate-resolution ( $R \geq 5000)$ observations of 50 quasars. Strong evolution of Ly $\alpha$ absorption at $z_{\text {abs }}>5$ is evident, the transmitted flux quickly approaches zero at $z>5.5$ (Figure 21). At $z_{\text {abs }}>6$, complete absorption troughs begin to appear: the Gunn-Peterson optical depths are $>>1$, indicating a rapid increase in the neutral fraction. However, is this evolution the result of a smooth transition due to gradual thickening of the Ly $\alpha$ forest (e.g. Songaila \& Cowie 2002, Songaila 2004)? Or is it a reflection of a more dramatic change in the IGM evolution, marking the end of reionization (e.g., Djorgovski et al. 2001, Becker et al. 2001, Fan et al. 2002)?

Djorgovski et al. (2001) detected an extended dark gap in the spectrum of SDSS J1044-0125 $(z=5.74)$, at $z_{\mathrm{abs}}=5.2-5.6$, with $\tau_{\mathrm{GP}}>4.6$. The first clearcut Gunn-Peterson trough was discovered in the spectrum of SDSS J1030+0524 ( $z=6.28$, Fan et al. 2001, Becker et al. 2001, Figure 4), which showed complete Gunn-Peterson absorption at $5.95<z_{\text {abs }}<6.15$ in both Ly $\alpha$ and Ly $\beta$ transitions. A high S/N spectrum presented in White et al. (2003) placed a stringent limit $\tau_{G P}>6.3$ in Ly $\alpha$. However, Songaila (2004) suggested that constraints based on Ly $\alpha$ alone do not deviate from a simple extrapolation from lower redshift by a large factor, consistent with a relatively smooth evolution in the ionization state.

The GP optical depth $\tau \propto f \lambda$, where $f$ and $\lambda$ are the oscillator strengh and rest-frame wavelength of the transition. For the same neutral density, the GP optical depth of $\operatorname{Ly} \beta$ and $\operatorname{Ly} \gamma$ are factors of 6.2 and 17.9 smaller than that of Ly $\alpha$. Therefore, Ly $\beta$ can provide more stringent constraints on the IGM ion- 
ization state when Ly $\alpha$ absorption saturates. Complete Ly $\beta$ absorption implies a significant rise in the neutral fraction towards SDSS J1030+0524. Songaila (2004) pointed out that in a clumpy IGM, the simple conversion factor of 6.2 between Ly $\alpha$ and Ly $\beta$ optical depths is an upper limit. Using high order Lyman lines requires proper treatment of the clumpy IGM. Oh \& Furlanetto (2005) and Fan et al. (2006b) found $\tau^{\alpha} / \tau^{\beta} \sim 2-3$ and $\tau^{\alpha} / \tau^{\gamma} \sim 4-6$ for a clumpy IGM.

Fan et al. (2006b) measured the evolution of Gunn-Peterson optical depths along the line of sight of the nineteen $z>5.7$ quasars from the SDSS (Figure 3). They found that at $z_{\text {abs }}<5.5$, the optical depth can be best fit as $\tau \propto(1+z)^{4.3}$, while at $z_{\text {abs }}>5.5$, the evolution of optical depth accelerates: $\tau \propto(1+z)^{>10}$. There is also a rapid increase in the variation of optical depth along different lines of sight: $\sigma(\tau) / \tau$ increases from $\sim 15 \%$ at $z \sim 5$, to $>30 \%$ at $z>6$, in which $\tau$ is averaged over a scale of $\sim 60$ comoving Mpc. Figure 4 (White et al. 2003) compares the Gunn-Peterson absorption in the two highest redshift quasars at the time of this writing: SDSS J1030+0524 $(z=6.28)$ shows complete absorption. On the other hand, SDSS J1148+5251 ( $z=6.42)$ shows clear transmission in Ly $\alpha$ and $\operatorname{Ly} \beta$ transitions at the same redshift range, clearly indicating that that this line of sight is still highly ionized (Oh \& Furlanetto 2005, White et al. 2005). The increased variance in the IGM optical depth at the highest redshifts is further discussed in Songaila (2004), Djorgovski et al. (2005) and Fan et al. (2006b).

Wyithe \& Loeb (2004) and Furlanetto \& Oh (2005) pointed out that at the end of reionization, the size of ionized bubbles grow rapidly. They estimate an observed bubble size of tens of Mpc at $z \sim 6$, and a scatter in the observed redshift of overlap along different lines-of-sight to be $\Delta z \sim 0.15$. Wyithe \& Loeb (2005a) further showed that the Gunn-Peterson optical depth variations to be of 
order unity on a scale of $\sim 100$ comoving Mpc. The increased variance on the IGM optical depth supports the interpretation that the IGM is close to the end of reionization.

With the emergence of complete Gunn-Peterson troughs at $z>6$, it becomes increasingly difficult to place stringent limits on the optical depth and neutral fraction of the IGM. Songaila \& Cowie (2002) suggested using the distribution of optically thick, dark gaps in the spectrum as an alternative statistic. Fan et al. (2006b) examined the distribution of dark gaps with $\tau>2.5$ among their sample of SDSS quasars at $z>5.7$, and showed a dramatic increase in the average length of dark gaps at $z>6$ (Figure [5), similar to the model prediction of Paschos \& Norman (2005). Gap statistics provides a powerful new tool to characterize the IGM ionization at the end of reionization (Gallerani et al. 2006, Kohler et al. 2006), and can be sensitive to larger neutral fractions, as they carry higher-order information than optical depth alone. Songaila \& Cowie (2002), Pentericci et al. (2002), Fan et al. (2002, 2006b) also studied using the distribution function of transmited fluxes (e.g. Rauch et al. 1997) or statistics of threshold crossing (e.g. Miralda-Escudé et al. 1996).

\subsection{Estimating the ionization state and the neutral fraction}

Measurements of Gunn-Peterson optical depth can be used to derive the ionization state of the IGM, using parameters such as the mean UV ionizing background, the mean-free-path of UV photons, and neutral hydrogen fractions. The evolution of Gunn-Peterson absorption is usually described in the context of photoionization. Weinberg et al. (1997) present the basic formalism to use the IGM optical depth to measure the cosmic baryon density. McDonald et al. (2000) and 
McDonald \& Miralda-Escude (2001) expanded this work to study the evolution of the ionizing background at $z<5.2$ by comparing the observed transmitted flux to that of artificial Ly $\alpha$ forest spectra created from cosmological simulations. Although slightly different in technical details, a number of works (Cen \& McDonald 2002, Fan et al. 2002, Lidz et al. 2002, Songaila \& Cowie 2002, Songaila 2004) followed the same formalism to calculate the evolution of the ionizing background at $z>5$ and reached consistent results: from $z \sim 5$ to $z>6$, there is an order of magnitude decrease in the UV background and the mean-free-path of UV photons is shown to be $<1$ physical Mpc at $z>6$ (Fan et al. 2006b). This scale is comparable to the clustering scale of star-forming galaxies at these redshifts (e.g. Kashikawa et al. 2005, Hu et al. 2005, Malhotra et al. 2005). High-redshift star forming galaxies, which likely provide most of the UV photons for reionization, are highly biased and clustered at similar physical scales to the mean free path. The assumption of a uniform UV background is no longer valid. There is a clear indication of a non-uniform UV background at the end of reionization (Fan et al. 2006b). However, current observations at $z>6$ are based on a handful of quasars. A much larger sample is needed to quantify the variation of the ionization state.

Using the same data, Fan et al. (2002, 2006b), Lidz et al. (2002), Cen \& McDonald (2002) find that at $z>6$ the volume-averaged neutral fraction of the IGM has increased to $>10^{-3.5}$. The results are displayed in Figure [ It is important to note that this is strictly a lower limit, due to the large optical depths in the Ly $\alpha$ line. Further, the presence of transmitting pixels and the finite length of dark gaps in the quasar spectrum can also be used to place an independent upper limit on the neutral fraction to be $<10-30 \%$ (Furlanetto et al. 2004, Fan 
et al. 2006b). At higher neutral fraction, the Gunn-Peterson damping wing from the average IGM (see §4.4) could wipe out any isolated HII region transmissions.

In summary, the latest work on GP absorption toward the highest redshift QSOs implies a qualitative change in the nature of $\operatorname{Ly} \alpha$ absorption at $z \sim 6$, including: (i) a sharp rise in the power law index for the evolution of GP optical depth with redshift, (ii) a large variation of optical depth between different lines of sight, and (iii) a dramatic increase in the number of dark gaps in the spectra. The GP results indicate that the IGM is likely between $10^{-3.5}$ and $10^{-0.5}$ neutral at $z \sim 6$. While saturation in the GP part of the spectrum remains a challenge, the current results are consistent with conditions expected at the end of reionization, during the transition from the percolation, or overlap, stage to the post-overlap stage of reionization, as suggested by numerical simulations (Paschos \& Norman 2005; Gnedin 2000, 2004; Ciardi et al. 2001, Razoumov et al. 2002, Gnedin \& Fan 2006). Sources at higher redshift, and estimators sensitive to larger neutral fractions, are needed to probe deeper into the reionization era. Wide-field deep near-IR surveys using dedicated 4-meter class telescopes, such as UKIDSS (Lawrance et al. 2006) and VISTA (McPherson et al. 2004), will likely discover quasars at $z \sim 8$ in the next decade (see also $§ 5.2$ for GRB observations).

\section{Cosmic Strömgren spheres and surfaces}

Luminous quasars produce a highly ionized HII region around them even when the IGM is still mostly neutral otherwise. The presence of (time bounded) cosmic Strömgren spheres (CSS) around the highest redshift SDSS QSOs has been deduced from the observed difference between the redshift of the onset of the GP

effect and the systemic redshift of the host galaxy (White et al. 2003; Wyithe \& 
Loeb 2004a; Wyithe et al. 2005; Walter et al. 2003). The physical size of these spheres is typically $\sim 5 \mathrm{Mpc}$ at $z>6$, or an order of magnitude larger volume than the typical spheres expected from clustered galaxy formation (Furlanetto, Zaldarriaga, \& Hernquist 2004), due to the extreme luminosity of the QSOs $\left(\sim 10^{14}\right.$ $\left.\mathrm{L}_{\odot}\right)$. The size of the Strömgren spheres is determined by the UV luminosity of the QSO, the HI density of the IGM, and the age of the QSO. Wyithe \& Loeb (2004a) and Wyithe et al. (2005) use QSO demographics, plus the distribution of CSS sizes, to infer a mean neutral IGM fraction of $x_{H I} \geq 0.1$ at $z \sim 6$.

A complementary analysis considers the 'cosmic Strömgren surface' around the $z=6.28$ QSO SDSS J1030+0524. Using high quality spectra (see Figure 4), Mesinger \& Haiman (2004) show that the apparent size of the CSS around this QSO is smaller using the $\operatorname{Ly} \alpha$ than the $\operatorname{Ly} \beta$ line. They attribute this difference to the damping wing of Ly $\alpha$, and from this infer an IGM neutral fraction $x_{H I} \geq 0.2$.

However, Oh \& Furlanetto (2004) highlight the many significant uncertainties in both these calculations, including: knowledge of the systemic redshift of the source, unknown 3D geometry, QSO age distribution, effect of large scale structure and clustering of ionizing sources around early luminous quasars, and often noisy spectra taken in a difficult wavelength range. In particular, recombinations in denser regions can be a significant photon sink, and hence the clumping factor becomes a key parameter. Yu \& Lu (2005) estimate that these factors could result in up to an order of magnitude uncertainity in the derived neutral fraction. Also, there remains debate as to whether these techniques measure a mass or a volume averaged neutral fraction. Fan et al. (2006b) show that over $5.7<z<6.4$, the average size of the quasar Strömgren spheres decreases by $\sim 2.5$, consistent with an increase of the neutral fraction by a factor $\sim 15$ over this redshift range, 
assuming the Strömgren radius $R_{s} \propto F(\mathrm{HI})^{-1 / 3}$ and other conditions being the same. Measurements of CSS are sensitive to much larger neutral fraction than using Gunn-Peterson effect, but are also strongly affected by systematics. More examples of $z>6$ QSOs over a larger range in intrinsic luminosity are required to verify these calculations.

\section{Other IGM Probes}

In this section, we describe other IGM probes that are either sensitive to a large neutral fraction, or could be extended to higher redshift in the near future.

\subsection{Evolution of metal absorption systems}

The early star formation that presumably started cosmic reionization also enriched the ISM and IGM with heavy elements. Detailed metal enrichment models in the early universe are reviewed in Ciardi \& Ferrara (2005). Songaila (2001, 2005) and Pettini et al. (2003) found that the total CIV abundance $\Omega(\mathrm{CIV})$ does not significantly evolve over $1.5<z<5.5$. Schaye et al. (2003) found a lower bound of $[\mathrm{C} / \mathrm{H}] \sim-4$ even in the underdense regions of the IGM at $z<4$. Madau et al. (2001, see also Stiavelli et al. 2004) found that by assuming massive stars ionized the Universe with yield from Population II or III stars, the Universe would have a mean metallicity of $\sim 10^{-3 \sim-4} Z_{\odot}$ after reionization, comparable to those found in the high-redshift quasar absorption lines. While these measurements do not directly constrain the reionization history, they suggest that one could detect numerous metal absorption lines even at $z>6$.

Furlanetto \& Loeb (2003) suggest using observations of low-ionization absorption lines such as CII or OI to constrain properties of the stellar population that 
ionized the Universe at $z>6$, such as star formation efficiency and escape fraction from supernova winds. To directly constrain the reionization history, Oh (2002) suggested using OI $\lambda 1302$ absorption as tracer of the neutral fraction. OI has almost identical ionization potentials to $\mathrm{H}$ and should be in tight charge exchange equilibrium with $\mathrm{H}$, while its lower abundance means that it would not saturate

even when the Universe was mostly neutral: $\tau_{\mathrm{OI}}^{\text {eff }}=10^{-6}\left(\frac{\langle Z\rangle}{10^{-2} Z_{\odot}}\right) \tau_{\mathrm{HI}}^{\text {eff }}$. OI (and $\operatorname{SiII} \lambda$ 1260) forests could provide combined constraints on the reionization and metal enrichment histories. Oh (2002) predicted that a handful of OI lines could be detected in the Gunn-Peterson trough redshift regions of known $z>6$ quasars when observed at high resolution. Becker et al. (2006) obtained high-resolution, high $\mathrm{S} / \mathrm{N}$ spectra of a sample of six quasars at $z>5$ using Keck/HERES and detected OI system up to $z=6.26$. They do not find a dense OI forest, consistent with high degree of IGM ionization at $z \sim 6$. However, it is puzzling that the line of sight of SDSS J1148+5251 $(z=6.42)$, which has the highest ionization fraction at $z>6(\S 3.3)$, also has the highest density of OI lines, raising the possibility that low metalicity, not high ionization, may be the cause for the lack of OI lines. Detailed modelling of IGM enrichment is needed to inteprete high-redshift metal line results.

\subsection{GRBs}

Gamma-Ray Bursts (GRBs) are the most powerful explosions in the Universe, and could be detected at $z>10$. At high-redshift, the time dilation means that their afterglow will fade away $(1+z)$ times slower, aiding rapid spectroscopic follow-up observations (e.g. Ciardi \& Loeb 2000) to probe the IGM evolution. GRB afterglow has been detected up to $z=6.30$ (SWIFT GRB 050904, Price et 
al. 2005, Tagliaferri et al. 2005, Haislip et al. 2005, Kawai et al., 2006).

For a largely neutral IGM, $\tau \sim 10^{5}$, the damping wing of the GP trough arising from the large GP optical depth of the neutral medium will extend into the red side of the Ly $\alpha$ emission line (Miralda-Escudé 1998). For $z \sim 6$, at $\sim 10 \AA$ redward of Ly $\alpha$ of the host galaxy, the optical depth is of order unity for a neutral IGM. However, this GP damping wing test cannot be applied to luminous quasars, due to the proximity effect from the quasar itself, as shown by Madau \& Rees (2000) and Cen \& Haiman (2000) and discussed in $\S 4$. Absorption spectra of GRBs, however, are not affected by the proximity effect and can be used to probe the existence of damping wings and measure IGM neutral fractions up to order of unity. However, strong internal absorption from the neutral hydrogen of the ISM $(\log \mathrm{N}(\mathrm{HI})>21)$ in the host galaxy appears to be ubiquitous among GRB afterglow spectra (e.g., Chen et al. 2005). Such internal absorption, or gas infall in the host galaxy environment (Barkana \& Loeb 2004), will complicate the interpretation of GRB observations. At large distance from line center, the damping wing from a diffused IGM has a profile $\tau \propto \Delta \nu^{-1}$, instead of $\tau \propto \Delta \nu^{-2}$ for a discrete absorber. Totani et al. (2006) fit a Ly $\alpha$ absorption profile of GRB $050904(z=6.30)$ with contributions from the internal damped Ly $\alpha$ absorption and the diffuse IGM damping wing simultaneously, and obtain a conservative limit on the IGM neutral fraction $x_{H I}<0.6$.

\subsection{Evolution of the IGM thermal state}

Reionization will photo-heat the IGM to several times $10^{4} \mathrm{~K}$. After this episode, the IGM will gradually cool mostly due to the Hubble expansion. Because of its long cooling time, the IGM will retain some of its thermal memory of reioniza- 
tion - earlier reionization leads to a cooler IGM at lower redshifts. Theuns et al. (2002a) used absorption line width measurements to estimate the IGM temperature at $z=2-4$, and found an average temperature of $\sim 2.5 \times 10^{4}$ at $z \sim 3.5$. This temperature constrains reionization to be: $z_{\text {reion }}<9$. Hui \& Haiman $(2003)$ carried out a similar analysis and considered different ionizing sources and different ionization histories. Figure 7 shows their models with different reionization redshifts and a quasar-like ionizing spectrum. They concluded that for all models where the Universe was ionized at $z>10$, and remained ionized thereafter, the IGM would have reached an asymptotic temperature too cold compared with observations. The IGM thermal history measurement requires the ionized fraction of the IGM to have of order unity changes at late $(6<z<10)$ epoch, regardless of whether there is a very early episode of reionization. This independent constraint on the reionization history is consistent with Gunn-Peterson measurements, and it points to a rapid ionization transition at low redshift. However, this is a difficult observation, and the interpretation of IGM temperture evolution may be further complicated by IGM heating during HeII reonization at lower redshift, (e.g., Sokasian et al. 2002).

\subsection{Luminosity function and line profiles of Ly $\alpha$ galaxies}

Surveys of galaxies with strong Ly $\alpha$ emission lines through narrow-band imaging in selected dark windows of the night sky $\mathrm{OH}$ emission forest have proven to be a powerful technique for discovering the highest redshift galaxies (e.g., Hu et al. 2002, 2004, Kodaira et al. 2003, Rhoads et al. 2003, Malhotra \& Rhoads 2004, Tran et al. 2004, Kurk et al. 2004, Santos et al. 2004, Martin \& Sawicki 2004, Taniguchi et al. 2005). At the time of this writing, $>100$ Ly $\alpha$ galaxies 
candidates have been found at $z \sim 6.5$, including $>30$ with spectroscopic confirmations, plus the most distant galaxy with a confirmed spectroscopic redshift, SFJ J132418.3+271455 at $z=6.589$ (Ajiki et al. 2003). Currently, ambitious surveys of $\operatorname{Ly} \alpha$ galaxies at even higher redshift, through windows in the near-IR, are underway (e.g. Barton et al. 2004, Horton et al. 2004).

Ly $\alpha$ galaxies represent a significant fraction of star forming galaxies at high redshift (Bouwens et al. 2006). Properties of Ly $\alpha$ galaxies directly probe the IGM neutral fraction. As described above, in a largely neutral IGM, the GunnPeterson damping wing extends to the redside of Ly $\alpha$ emission. Without a large Strömgren sphere, the intrinsic Ly $\alpha$ emission will be considerably attenuated. In the simplest picture, one predicts: (1) the Ly $\alpha$ galaxy luminosity function will decrease sharply in an increasingly neutral IGM, even if the total star formation rate in the Universe remains roughly constant, and (2) the Ly $\alpha$ profiles will have a stronger red wing and a smaller average equivalent width before the onset of reionization.

Malhotra \& Rhoads (2004) and Stern et al. (2004) combined the LALA survey of Ly $\alpha$ galaxies (Rhoads et al. 2003) with other Ly $\alpha$ surveys in the literature to determine the luminosity function of $\operatorname{Ly} \alpha$ galaxies at $z=6.5$ and 5.7. They found no evolution between these two redshift bins, consistent with the IGM being largely ionized by $z \sim 6.5$ (Figure 8 ). Hu et al. (2005) constructed Ly $\alpha$ line profiles at $z=6.5$ and 5.7 from their surveys using Keck and Subaru, and found a similar lack of evolution. The interpretations of these results, however, require more detailed modelling. Haiman (2002), Santos (2004), Cen et al. (2005) showed that the local HII regions around Ly $\alpha$ galaxies reduce the attenuations of Ly $\alpha$ flux. Gnedin \& Prada (2004), Wyithe \& Loeb (2005b), Cen (2005) and Furlanetto 
et al. (2005) further demonstrated that the clustering of ionizing sources increases the HII region size and further reduces the attenuation. Haiman \& Cen (2005) estimated that the lack of evolution in the Ly $\alpha$ luminosity function is consistent with the neutral fraction $x_{H I}<0.25$, when no clustering is considered (see also Malhortra \& Rhoads 2005). Including large scale clustering, the constraint on the IGM neutral fraction becomes less stringent (Furlanetto, Zaldarriago \& Hernquist 2005). Santos (2004) showed that the presence of galactic winds may also play a crucial role in determining the observed Ly $\alpha$ fluxes. Haiman \& Cen (2005) suggest studies of $\operatorname{Ly} \alpha$ profiles as a function of luminosity, compared with lowredshift samples, could provide robust diagnostics to the IGM ionization state. Furlanetto et al. (2005) showed that at large neutral fraction, only galaxies in large ionized bubbles can be detected in $\operatorname{Ly} \alpha$, which have larger bias than individual galaxies. With future large area $\operatorname{Ly} \alpha$ surveys, it may be possible to measure the size distribution of HII regions during reionization in order to constrain reionization history.

\section{Cosmic Microwave Background Probes of Reionization}

The Cosmic Microwave Background (CMB) is a rich "fossil record" of the early universe. The CMB not only reveals the universe's initial conditions, but also its structure and dynamics from the time of the CMB's origin 400,000 years after the Big Bang to today. A CMB photon arriving in a terrestrial, balloonborne, or space-based CMB observatory is encoded with the conditions of the universe along its 13.7 billion year journey. In particular, since the CMB is the oldest observable electromagnetic radiation it acts as a backlight, illuminating the transition from bulk-neutrality to complete reionization. Both the temperature 
anisotropy and polarization of the CMB help reconstruct the details of the EoR. As we will see, the CMB's polarization and temperature anisotropy are completely complementary probes; both in their spatial structure, and in their dependence on the conditions of the EoR which they probe.

\subsection{The Generation of CMB Polarization and Temperature Anisotropy During the EoR}

Thomson scattering produces CMB polarization only when free-electron scatterers are illuminated by an anisotropic photon distribution ( $\mathrm{Hu} \&$ White(1997)) Moreover, the photon anisotropy, when decomposed into spherical harmonics $Y_{\ell, m}$ in the rest frame of the electron, must possess a non-zero quadrupole $(\ell=2)$ term - no other contributes due to the orthogonality of the spherical harmonics. Both free-electrons and anisotropically distributed photons were present during the transition from complete ionization to the EoR. This epoch produced the primary CMB polarization signal, referred to as "E-mode" or "gradient mode" owing to its symmetry properties under parity transformations (Zaldarriaga \& Seljak(1997), Kamionkowski et al.(1997)]. The anti-symmetric component of CMB polarization is referred to as "B-mode" or "curl mode" and arises in inflationary cosmological models which predict a primordial gravitational wave background. The E-mode polarization anisotropy is produced by the same perturbations which produce temperature anisotropy and peaks at $\simeq 10^{\prime}$ angular scales (or multipoles $\ell \simeq 1000$ ) with an amplitude approximately $10 \%$ of the CMB temperature anisotropy at large $\left(>10^{\circ}\right)$ angular scales.

We begin this section by briefly reviewing how the EoR produces new CMB polarization and temperature anisotropy. The physics of CMB polarization and 
anisotropy has been treated in numerous sources. We refer the reader to (Zaldarriaga \& Selja Kamionkowski et al.(1997) Hu \& White(1997) Dodelson(2003) for analytic and theoretical treatments and (Seljak \& Zaldarriaga(1996) $)$ for numerical calculations. Ultimately, the CMB data, in combination with the $21 \mathrm{~cm}$ emission and Ly $\alpha$ absorbtion by neutral HI, will allow for a detailed reconstruction of the physics of the EoR.

\subsubsection{Large Angular Scale CMB Polarization and Reionization} Large-angular scale CMB polarization as a probe of the ionization history of the universe, has been considered in e.g. (Zaldarriaga(1997), Keating et al.(1998), Kaplinghat et al.(2003)). The importance of large-angular scale CMB polarization is that, unlike the $21 \mathrm{~cm}$ and Ly $\alpha$ HI-absorption measurements discussed elsewhere in this review, the polarization of the CMB is sensitive to ionized hydrogen (HII) as it is generated by Thomson scattering.

Reionization produces free-electrons which Thomson-scatter CMB photons, producing CMB polarization. For angular scales smaller than the horizon at reionization the scattering damps the CMB temperature in direction $\hat{\mathbf{n}}$ as $T^{\prime}(\hat{\mathbf{n}})=$ $e^{-\tau} T(\hat{\mathbf{n}})$, where $\tau$ is the Thomson optical depth. Reionization, therefore, damps the temperature anisotropy power angular spectrum as $C_{\ell}^{T^{\prime}}=e^{-2 \tau} C_{\ell}^{T}$. The damping of the temperature power spectrum is degenerate with the primordial power spectrum's amplitude, $A$, for scales smaller than the horizon at last scattering.

Fortunately, a new feature, e.g. (Zaldarriaga(1997)), in the E-mode power spectrum develops at large angular scales which breaks the degeneracy between $A$ and $\tau$. The degeneracy (for the CMB temperature anisotropy) and its breaking (using CMB polarization) is illustrated in (real-space) in figure 9, Figure 10 shows 
the polarization angular power spectrum associated with these simulations, along with current observational results (discussed further in 6.2). Note in 10 the new large-angular scale (low- $\ell$ ) peak which discriminates between models with and without reionization.

The polarization anisotropy of the CMB reveals the ionization condition of the universe in ways which the temperature anisotropy cannot. Following recombination at $z=1089 \pm 1(\underline{3})$ primordial hydrogen remained neutral until the epoch of reionization. The term "last-scattering" is only approximately true since we know that the universe is nearly $100 \%$ ionized at present $(z=0)$. Therefore some fraction (few to $\sim 10 \%$ ) of the photons scattered again following decoupling. If reionization was instantaneous, only the primordial temperature quadrupole at the "last-scattering surface" $(z \simeq 1100)$ projected to the redshift of reionization contributes to the $\mathrm{CMB}$ polarization at the $<1 \mu \mathrm{K}$ level (at $>10^{\circ}$ scales). This reionization feature has been detected by WMAP and is interpreted as a detection of $\tau=0.10 \pm 0.03$, or $\tau=0.09 \pm 0.03$ when all six cosmological parameters are fit to all of the WMAP data (TT,TE,EE)(page) .

Whereas CMB temperature anisotropy is generated by processes occurring after reionization (e.g., gravitational redshifts due to matter inhomogeneity along the line of sight - the Integrated Sachs-Wolfe (ISW) effect), the large-scale polarization of $\mathrm{CMB}$ is not similarly affected. The large-scale CMB polarization is a cleaner and more precise probe of the onset of the reionization epoch than the anisotropy for two reasons. The first is that the ratio of the polarized intensity to the total intensity is unchanged when the CMB propagates through an absorbing medium (such as the post-reionization intergalactic plasma) - both polarization components are attenuated equally. The second is that both polarization 
components incur equal gravitational redshifts (blueshifts) when climbing out of (falling into) time-varying gravitational potential wells from last-scattering to today since gravity cannot distinguish between the two linear polarization states. This is equivalent to asserting that CMB polarization does not experience the ISW effect, and so the low-multipole E-mode spectrum is not contaminated by the ISW effect.

High precision observations of the large-scale polarization will reveal more of the EoR's details. The polarization power spectra are sensitive to the duration of reionization. With upcoming observations the dynamics of the EoR can be constrained, complementing $21 \mathrm{~cm}$ observations (§8), by providing (modest) redshift information; most notably regarding the transition from partial to total reionization by constraining $x_{H I}(z)$ - difficult using $21 \mathrm{~cm}$ data alone. This would be a powerful probe of "double reionization" models(?). A cosmic variance limited experiment, with sufficient control over instrumental systematics and foregrounds, can distinguish the transition from partial to total reionization using details of the polarization power spectra (Kaplinghat et al.(2003), Holder et al.(2003)). Kaplinghat et al. show that large-scale CMB polarization can constrain the redshift of the transition from partial to total reionization with precision $\sigma_{z_{r i}}=333 \sigma_{\tau} / \sqrt{z_{r i}}$, where $\sigma_{\tau}$ is the precision with which the experiment measures the optical depth. Upcoming measurements, such as Planck, will produce percent-level constraints on partial reionization or double reionization scenarios and discriminate between models with identical $\tau$, but very different ionization histories (Kaplinghat et al.(2003)).

Reionization also produces a new low- $\ell$ peak in the B-mode polarization pattern, which is absent in models without reionization. Even a modest ionization 
fraction increases the observability of the B-mode (curl-mode) polarization. This is significant because gravitational lensing of large scale structure converts Emode power into B-mode power (Zaldarriaga \& Seljak(1998) Hu \& Okamoto(2002)). This contaminant increases with increasing multipoles up to $\ell \sim 1000$. With the new, low- $\ell$ structure, a more stringent limit on the inflationary-generated gravitational wave background can be obtained than would be possible without reionization( Kaplinghat, Knox and Song (2003) $)$.

\subsubsection{Small Angular Scale CMB Polarization and Reionization}

Because even the primary CMB polarization is so small, observing effects to second order in $\tau$ or distinguishing the details of the Strömgrensphere percolation process is extremely challenging using CMB polarization. Fortunately, the small angular scale CMB anisotropy will provide a rich data set with vital information on the fine-details of the reionization epoch.

\subsubsection{THE EFFECTS OF REIONIZATION ON CMB TEMPERATURE ANISOTROPY} CMB polarization probes the onset of reionization and distinguishes partial reionization from complete, but it cannot probe the details of the ionization percolation process as it is sensitive to the integrated Thomson optical depth to the last scattering surface. In fact, the reionization details which can be provided by large scale polarization is known to be limited by cosmic variance ( $\mathrm{Hu} \&$ Holder 2003) and the smoothing inherent in large scale polarization measurements. Fortunately, the CMB temperature anisotropy at small angular scales may reveal many of these interesting features (?, Gruzinov \& Hu (1998), Knox et al.(1998), Haiman \& Knox(1999) Barkana \& Loeb 2001 Santos et al. 2003 Zahn et al.(2005)).

Thus, both the bulk and fine details of reionization will be probed by the complementary measurements of large-scale CMB polarization anisotropy and small- 
scale temperature anisotropy observations.

In non-instantaneous models of reionization (due to discrete ionizing sources), reionization proceeds in a "patchy" manner where the Strömgrenspheres of ionized hydrogen surrounding the sources eventually coalesce to produce the fullyionized universe observed out to $z \sim 6$. This patchiness induces secondary CMB temperature anisotropy (?,Gruzinov \& Hu (1998) Knox et al.(1998) McQuinn et. al. (2005 In addition to the damping of the CMB temperature anisotropy power spectrum, there are two interesting secondary temperature anisotropy effects: 1) the kinetic Sunyaev-Zel'dovich effect (kSZ) and 2) the Ostriker-Vishniac effect (OV).

The kSZ effect is due to the motion of regions of reionized electrons along the line of sight. It is similar to the kinematic SZ effect produced by inverse Compton scattering of the CMB by electrons in moving galaxy clusters (Sunyaev \& Zeldovich(1980)), but here it refers to the motions of the ionized electrons in the pre-galactic medium (PGM) during the EoR. At very small angular scales $\left(<4^{\prime}\right)$ the primary CMB anisotropy signal is damped due to photon diffusion out of overdense regions (Silk damping) prior to decoupling. Here the thermal SZ effect dominates over the primary CMB anisotropy, but at the thermal SZ null $(218 \mathrm{GHz})$ the EoR $\mathrm{kSZ}$ effect may be the dominant source of CMB temperature anisotropy.

During reionization, electron spatial inhomogeneity can arise in two ways: either by a variable ionization fraction, $x_{i}$, of by a constant $x_{i}$ and a variable baryon density. This effect is the OV effect which is primarily generated by structures in the non-linear regime, $\underline{\text { i.e., }}$ at low- $z$. The OV effect is the result of a "patchy" reionzed medium. Much progress has been made due to the development of fast structure formation codes (e.g., smooth particle hydrodynamics - SPH) which trace the reionization mechanism (since they trace the formation of baryonic 
structures). The reionization efficiency of the baryonic structures is the only free parameter, and many of these approaches, e.g., Zahn et al. (2005) incorporate semi-analytic reionization models into their simulations. Using an extended Press-Schechter formalism to model the reionization process Zahn et al. (2005) find that patchy reionization makes a large contribution to the CMB anisotropy at scales of $6^{\prime}(\ell \sim 2000)$ which exceeds the kSZ effect. In fact, ignoring these secondary, EoR effects will lead to significant biases in the estimation of cosmological parameters derived from the primary CMB anisotropy, which further underscores vital importance of their measurement (Santos et al. 2003, Zahn et al.(2005)).

Both the $\mathrm{kSZ}$ effect and the OV effects occur at small scales $\left(<0.1^{\circ}, \ell>\right.$ 2000) and probe both the homogeneity and efficiency of the reionization process. The shapes of the predicted temperature anisotropy power spectra for these secondary effects appear to be robust to changes in the reionization model (Knox(2003), Santos et al. 2003, Zahn et al.(2005)), though the peak-position of the secondary power spectra occurs when $x_{H I} \sim 0.5$, which is model-dependent (Furlanetto et al. 2005a). In contrast, the overall amplitude of the secondary spectra appears to be significantly model-dependent. However, the amplitude depends only weakly on the epoch of reionization and more strongly on the duration of the patchy phase, making it complementary to the CMB polarization measurements of the optical depth(McQuinn et. al. (2005)). For completeness we close this subsection on secondary effects by mentioning that both the polarized kSZ and OV effects are expected to be nearly negligible and practically impossible to measure (Seshadri \& Subramanian (1998), $\mathrm{Hu}(2000)$ 


\subsection{Current CMB Results and Future Prospects}

The first detection of reionization phenomena using CMB observations came with NASA's Wilkinson Microwave Anisotropy Probe (WMAP) which reported a nearly $5 \sigma$ detection of a reionization feature in the large angular scale $\left(>10^{\circ}\right)$ temperature-polarization cross-correlation function $(\langle T E\rangle)$ with only a single year of data(Kogut et al.(2003)). This detection was the first use CMB polarization to measure the physics of the EoR. It also was the first detection of the Thomson optical depth, $\tau$, rather than the lower limits (provided by Ly $\alpha$ measurements) or upper limits (provided by CMB temperature anisotropy measurements). WMAP's initial detection of $\tau$ in 2003 using the TE-data and subsequent detection using the E-mode polarization only (page) also determines the primordial power spectrum amplitude $A, \sigma_{A} \simeq 2 \sigma_{\tau}$ (3). WMAP's detection illustrates CMB polarization's power to probe vitally important cosmological parameters that are essentially unobservable using temperature anisotropy alone.

WMAP's three-year data set (page) reports several detections of the E-mode and temperature-polarization cross-correlation power spectrum, especially at low$\ell$ (due to WMAP's ability to map most of the sky). CBI, DASI, and BOOMERANG(Piacent have also detected the $\langle T E\rangle$ cross-correlation spectrum, at smaller angular scales than WMAP. WMAP's first-year results are relatively robust to the choice of additional data sets and priors used to constrain cosmological parameters (including $\tau$ ), such as SDSS ((Tegmark et al.(2004))) and Ly $\alpha$ forest measurements (Seljak et al.(2005)).

In figures 10 and11 observational CMB polarization and temperature-polarization cross-correlation power spectra are shown for the angular scales relevant for probing the EoR. The primary effects of reionization are encoded in the E- 
mode autocorrelation and temperature polarization cross-correlation power spectra at $\ell \lesssim 50$. As demonstrated in, e.g., (Zaldarriaga(1997), Hu \& Holder 2003, Keating \& Miller (2005) ) essentially all the information on $\tau$ comes from $\ell<50$. While most of the $\tau$-constraint comes from $\ell<10$, a non-negligible amount comes from $10<\ell<40$. Currently, only WMAP(page) and BOOMERANG(Montroy et al.(2005)) have detected E-mode polarization at angular scales greater than $1^{\circ}(\ell<200)$, and only WMAP has detected the E-mode polarization for $\ell<100$. CBI(Readhead et al.(2004) and CAPMAP(Barkats et al.(2005) have detected the E-mode polarization, though at smaller angular scales (higher $\ell$ ) than are relevant for probing the EoR with CMB polarization alone.

While no detections of the $\mathrm{kSZ}$ or OV effects have been reported, several groups have published detections of CMB anisotropy at scales which are relevant to both secondary temperature effects. For $3000<\ell<10000$, the kSZ temperature anisotropy power spectrum scales roughly as $C_{l}\left(\Omega_{b} h\right)^{2} \sigma_{8}^{p}$ where $3<p<7$ (see e.g. (Zhang et al.(2004)) ). (Dawson et al.(2002)) report a detection of CMB anisotropy at sub-arcminute $(\ell \simeq 7000)$ scales using the BIMA array and (Readhead et al.(2004b) report a detection at larger (few-arcminute) scales using CBI. The results of the two groups are consistent, and seem to indicate significant power in excess of the CMB primary anisotropy at those scales. Both groups speculate on the likelihood that this excess structure is due to the thermal SZ effect. While neither group has detected the kSZ or OV effects, they are valuable technological and methodological precursors for future CMB anisotropy measurements of the EoR. 


\subsection{Observational Challenges}

A hallmark of CMB experiments since the detection of the CMB has been the mitigation and excision of systematic effects. CMB observations have gone to great lengths to combat systematic effects, including building duplicate telescopes (e.g. the COBE and WMAP satellites). Another method of improving experimental fidelity is to conduct extremely wide-band observations for foreground monitoring and removal. For example, WMAP has radiometers covering more than two octaves in frequency(Jarosik et al.(2003)), and Planck's radiometers will cover nearly five octaves(Lamarre et al.(2003), Mennella et al.(2004)).

Particularly for the large scale E-mode polarization, measuring the CMB with multiple frequency channels alone is not sufficient. To overcome statistical (cosmic) variance on the low- $\ell C_{\ell}^{E}$ peak, an experiment must observe a large survey region - at least $\sim 30^{\circ} \times 30^{\circ}$. Such a large area is likely to be contaminated by galactic emission, which can only be subtracted to finite precision. The most conservative approach to deal with galactic foregrounds is to exclude regions of strong foreground emission (such as the galactic plane) from the survey. For example, the WMAP team used multiple frequency channels in combination with sky-cuts that removing $15 \%$ of the sky surrounding the galactic plane (Kogut et al.(2003) and introduced correlations between multipoles at the few percent-level. Even with sophisticated foreground modelling and multiple frequency coverage the challenges of achieving polarization fidelity at the $100 \mathrm{nK}$ level over $>10^{\circ}$ angular scales - required, for example, to detect the signatures of non-instantaneous reionization scenarios (e.g, (Kaplinghat et al.(2003) Holder et al.(2003) ) - are daunting. The CMB community is well aware of the challenge; see for example, the CMB Task Force Committee report (Weiss et al.(2005)). 
Observational challenges for secondary temperature anisotropy (kSZ and OV) measurements include point sources, primary CMB confusion, secondary CMB confusion due to gravitational lensing and the thermal SZ effect. To detect the secondary effects, the primary CMB anisotropy must be overcome by measuring at scales smaller than the Silk damping scale, requiring large ( $>5 \mathrm{~m}$ class) telescopes. Of the foregrounds for the $\mathrm{kSZ}$ and OV effects, the thermal SZ effect is perhaps the most straightforward to mitigate - by measuring at the thermal SZ-null near $218 \mathrm{GHz}$. At small scales, point sources need to be excised based on ancillary measurements of their spectra and position. There are also significant hurdles to overcome in the theoretical modelling of the secondary temperature effects. In particular, separating the low- $z$ OV effect from high- $z$ patchy reionization effects will be challenging, though hopefully amenable to theoretical modelling and simulation.

Since galactic foregrounds and point sources contribute to spurious polarization and anisotropy with different systematics, combining the large angular CMB polarization observations with the small scale temperature anisotropy measurements represents the most promising avenue towards a faithful reconstruction of the EoR using the CMB.

\subsection{Discussion and Future Prospects}

WMAP's on-going measurements of CMB polarization are expected to continue until at least 2007. Planck is expected to be launched in 2008 and will start making full-sky observations of the polarization and primary temperature anisotropy of the CMB. Planck is expected to achieve a nearly cosmic variance limited measurement of $\tau$. A balloon-borne CMB polarimeter called EBEX (Oxley et al.(2005) 
will cover a large region of the sky in an attempt to measure the signature of gravitational waves imprinted on the CMB.

More reionization information will emerge from CMB B-mode polarization observations, which search for the imprint of primordial gravitational radiation on the CMB. Like the E-mode polarization reionization signature, the B-mode polarization peaks at super-degree scales so the upcoming experiments optimized to search for the B-mode signal will have appreciable sensitivity to reionization features in the E-mode spectrum (Keating \& Miller (2005)).

An ancillary benefit of reionization is that it boosts the primary (inflation generated) B-mode power spectrum significantly near $\ell=10$. Due to reionization, a tighter limit on the tensor-to-scalar ratio, $r$, in the presence of gravitational lensing (at redshifts $z<10$ ) can be obtained than that calculated in Knox \& Song(2002) Kesden et al.(2002)). Kaplinghat, Knox \& Song (Kaplinghat, Knox ar demonstrate that the minimum detectable inflationary energy scale behaves as $1 / \tau^{4}$ due to the low- $\ell$ B-mode power spectrum peak produced by reionization. This motivates very wide-field, or full-sky, observations of CMB polarization. NASA's Beyond Einstein initiative features a CMB polarimeter called "CMBPol" ${ }^{1}$ This instrument is designed to detect the signature of inflationary gravitational waves over a wide range of inflationary energy scales. This experiment will achieve cosmic variance limited precision on $\tau$, which is essentially the same as Planck's sensitivity (Kaplinghat et al.(2003)), but in contrast to Planck, CMBPol will be optimized to detect CMB polarization.

Three large-format bolometric array telescopes are currently being developed to probe CMB temperature anisotropy and the kSZ and OV effects. The Atacama ${ }^{1}$ http://universe.nasa.gov/program/inflation.html 
Cosmology Telescope (ACT, $(\overline{\operatorname{Kosowsky}(2003)}))$ is a $6 \mathrm{~m}$ telescope operating in three frequency bands $(145,225$, and $265 \mathrm{GHz})$ with $\simeq 1^{\prime}$ angular resolution. With concordance cosmological parameters, ACT should be able to constrain the redshift of reionization to percent-level accuracy (Zhang et al.(2004)). The South Pole Telescope (SPT, Ruhl et al. 2004) is a $10 \mathrm{~m}$ telescope operating in 5 bands from 100 to $345 \mathrm{GHz}$ with angular resolution ranging from $1.5^{\prime}$ to $\simeq 0.5^{\prime}$. Finally, the largest CMB anisotropy telescope which will be sensitive to secondary CMB temperature anisotropy is the $12 \mathrm{~m}$ Atacama Pathfinder EXperiment (APEX) which will use a 320 pixel array of transition edge sensor (TES) bolometers, also from a high altitude ( $5000 \mathrm{~m}$ ) observatory in the Chilean Atacama desert. All of these instruments, with the exception of CMBPol, are scheduled for "first-light" before 2010 .

This section has emphasized the role of the CMB in illuminating the physics of the EoR. Both the CMB's polarization and its temperature anisotropy provide a wealth of reionization data in a fashion that is complementary to the $21 \mathrm{~cm}$ and Ly $\alpha$ observations discussed elsewhere in this review. While the importance of large-scale CMB polarization to reionization was recognized early-on, its ability to reveal more than the bulk Thomson optical depth was not initially appreciated. Now we understand that the polarization and the temperature anisotropy provide a detailed view of the EoR. In fact, they do so in completely complementary ways: the polarization reveals the epoch of reionization in a way the temperature anisotropy cannot. Likewise, the CMB's temperature anisotropy probes secondary effects to which the polarization is blind. When the new CMB polarization and temperature anisotropy observations are combined a high-fidelity image of the EoR will emerge. 


\section{Sources of Reionization}

Regardless of the detailed reionization history, the IGM has been almost fully ionized since at least $z \sim 6$. This places a minimum requirement on the emissivity of UV ionizing photons per unit comoving volume required to keep up with recombination and maintain reionization (Miralda-Escudé et al. 2000):

$$
\dot{\mathcal{N}}_{\text {ion }}(z)=10^{51.2} \mathrm{~s}^{-1} \mathrm{Mpc}^{-3}\left(\frac{C}{30}\right) \times\left(\frac{1+z}{6}\right)^{3}\left(\frac{\Omega_{b} h^{2}}{0.02}\right)^{2},
$$

where $C \equiv \frac{\left\langle n_{H}^{2}\right\rangle}{\left\langle n_{H}\right\rangle^{2}}$ is the clumping factor of the IGM. It is difficult to determine $C$ from observations, and has large uncertainty when estimated from simulations $(C=10-100$, Gnedin \& Osteriker 1997). At $z<2.5$, the UV ionizing background is dominated by quasars and AGN (e.g. Haardt \& Madau 1996). At $z>3$, the density of luminous quasars decreases faster than that of star-forming galaxies, and the ionizing background has an increased contribution from stars (e.g. Haehelt et al. 2001). In this section we summarize what is known about the ionizing background contribution from quasars and AGN, young stars, or other sources of high energy photons (e.g. particle decay).

\subsection{Quasars and AGN}

Quasars and AGN are effective emitters of UV photons. The UV photon escape fraction is generally assumed to be of order unity. The UV photon emissivity from quasars and AGN can be directly measured from integrating quasar luminosity functions at high-redshift. The density evolution of luminous quasars $\left(M_{1450}<\right.$ -27) has been well-determined from surveys such as SDSS (e.g. Warren et al. 1994, Kennefick et al. 1995, Schmidt et al. 1995, Fan et al. 2001a, b, 2004, Richards et al. 2005) up to $z \sim 6$. Luminous quasar density declines exponentially 
towards high-redshift: it is $\sim 40$ times lower at $z \sim 6$ than at its peak at $z \sim 2.5$.

However, quasars have a steep luminosity function at the bright end - most of the UV photons come from the faint quasars that are currently below the detection limit at high-redshift.

Fan et al. (2001b) showed that quasars could not have maintained IGM ionization at $z \sim 6$, as far as the shape of the luminosity function at $z \sim 6$ is not much steeper than that at $z<3$. In fact, Fan et al. (2001a) and Richards et al. (2005) used the SDSS sample to find that at least the bright end slope of quasars at $z>4$ are considerably flatter than that at low-redshift, consistent with the findings of the COMBO-17 survey (Wolf et al. 2003). Miralda-Escudé (2003), Yan \& Windhorst (2004a) and Meiksin (2005) used different parameterizations of quasar luminosity function evolution and came to the same conclusion. Willott et al. (2005) and Mahabal et al. (2005) present surveys of a few $\operatorname{deg}^{2}$ for faint $z \sim 6$ quasars. Their detection of only one $z \sim 6$ faint QSO implies that the quasar population contribution to the ionizing background is $<30 \%$ that of star-forming galaxies.

\subsection{Lyman Break galaxies}

Due to the rapid decline in the AGN populations at very high $z$, most theoretical models assume stellar sources reionized the universe. However, despite rapid progress, there is still considerable uncertainty in estimating the total UV photon emissivity of star-forming galaxies at high-redshift. Modifying the constraint from Equation 4, Bunker et al. (2004) and Bouwens et al. (2005) showed:

$$
\dot{\rho_{*}} \approx\left(0.026 \mathrm{M}_{\odot} \mathrm{yr}^{-1} \mathrm{Mpc}^{-3}\right)\left(\frac{1}{f_{\text {esc }, \mathrm{rel}}}\right) \frac{C}{30}\left(\frac{1+z}{7}\right)^{3} .
$$


where $\dot{\rho}_{*}$ is the UV star formation rate rate, $C_{30}$ is the IGM clumping factor, $f_{\text {esc,rel }}$ is the fraction of ionizing radiation escaping into the intergalactic medium to that escaping in the $U V$-continuum $(\sim 1500 \AA)$. The uncertainties come from three factors: the star formation rate, clumping factor, and UV escape factor. Current estimates of the star formation rate at $z \sim 6$ all come from photometrically-selected $i$-dropout objects selected in a small number of deep fields observed by the HST: the HUDF (Beckwith et al. 2005 in preparation), GOODS fields (Giavalisco et al. 2004), and UDF ACS Parallels (Thompson et al. 2005). These estimates are affected strongly by sample (or cosmic) variance at $z \sim 6$ due to the small volume of deep surveys, as well as estimates or extrapolations towards faint luminosities. The clumping factor is typically taken from cosmological simulations (e.g. $C \sim 30$, Gnedin \& Osteriker 1997). The UV escape fraction remains problematic. Direct measurements of the escape fraction range from upper limits, $<0.1$ to $<0.4$ (e.g. Giallongo et al. 2002, Fernández-Soto et al. 2003, Inoue et al. 2005) to $>0.5$ (Steidel et al. 2001). Therefore, instead of measuring the accurate UV photon emissivity, most efforts have been trying to determine whether star-forming galaxies could provide sufficient photons to at least meet the requirement in Equation 5 at $z \sim 6$.

Using HUDF data, Yan \& Windhorst (2004b) found 108 plausible candidates at $5.5<z<6.5$ down to $m_{\mathrm{AB}}(z)=30.0 \mathrm{mag}$. They estimated a steep faintend luminosity function with a power-law index of -1.8 to -1.9 , and concluded that this steep slope is sufficient to satisfy the reionization requirement, and that most of the photons that ionized the universe come from dwarf galaxies. Similarly, Stiavelli et al. (2004) compared GOODS and HUDF observations and found that the observed mean surface brightness of galaxies at $z \sim 6$ is sufficient 
for reionization when the young stellar population has a top heavy IMF. Bunker et al. (2004), however, found a much lower star formation density at $z \sim 6$ from the HUDF. Their results showed a factor of 6 decline in star formation rate from $z \sim 3$ to 6 . Even using the most optimistic escape faction $(f=1)$, their results imply insufficient photons to ionize the Universe by $z \sim 6$ from stellar sources.

Bouwens et al. (2005) combined all the available datasets that include 506 $i$-dropout $(z \sim 6)$ galaxy candidates to construct a luminosity function. Figure 12 summarizes the determination of the evolution of star-formation rate at both the luminous and faint end. The star formation rate begins to decline modestly at $z>5$. The shape of the luminosity function has also evolved significantly from $z \sim 3$ to 6 . However, the integrated UV luminosity (down to $0.04 L_{z=3}^{*}$ )

did not evolve significantly. Their results support the previous claim that dwarf galaxies provide the majority of ionizing photons at $z \sim 6$, sufficient to ionize the Universe.

Most recently, Kashlinsky et al. (2005) have reported the detection of a significant excess in the Spitzer IRAC bands above that expected based on known galaxy counts. They interpret this excess as being due to population III stars forming at $z>10$. These stars would clearly contribute to early reionization, although the magnitude of the contribution remains uncertain due to the difficulty of the measurement, and the uncertainty of the origin of this mid-infrared background.

\subsection{Dusty and big galaxies at high redshift}

Recent observations have revealed two surprising types of galaxies at $z>6$. First, millimeter observations of the host galaxies of the highest redshift QSOs 
reveal large dust masses $\left(\sim 10^{8} \mathrm{M}_{\odot}\right)$ in $\sim 30 \%$ of the sources (Bertoldi et al. 2003a). And in one case, J1148+5251 at $z=6.42$, millimeter line emission from CO has been detected, indicating a large molecular gas mass $\left(\sim 2 \times 10^{10} \mathrm{M}_{\odot}\right.$; Walter et al. 2003, 2004; Bertoldi et al. 2003b). Second, Eyles et al. (2005) and Mobasher et al. (2005) present Spitzer observations of $z \sim 6-6.5$ galaxies. These objects show SEDs characteristic of post-starburst galaxies, with stellar masses between $10^{10}-10^{11} M_{\odot}$ and stellar ages of several hundred Myrs. Reddening is also infered by Chary, Stern, \& Eisenhardt (2005) in one galaxy, implying a dusty galaxy $\left(A_{v} \sim 1\right)$. These results require active star formation $\left(>10^{2} M_{\odot}\right.$ year $\left.^{-1}\right)$ starting at $z \sim 8-15$. These objects are capable of ionizing large volumes at high-redshift, and could have played an important role in the early reionization of the IGM, depending on their currently ill-constained space density (Panagia et al. 2005; Barkana \& Loeb 2005c).

\subsection{X-ray photons and decaying sterile neutrios}

High-energy X-ray photons from mini-quasars or supernova remnants at highredshift could also contribute significantly to the reionization process (Oh 2001; Venkatesan et al. 2001; Madau et al. 2004; Ricotti \& Ostriker 2004). These photons have long mean-free-paths, and could pre-ionize the IGM significantly, providing the optical depth required by WMAP polarization measurement, and produce a rapid ending of reionization at $z \sim 6$ as suggested by the Gunn-Peterson measurements. However, Dijkstra et al. (2004) and Salvaterra et al. (2005) show that the hard X-rays from these same sources would produce a present-day soft Xray background. They calculate the X-ray background if such photons dominated the reionization budget, and conclude that models with accreting black holes (a 
combination of luminous and faint AGN) would overproduce the observed X-ray background by a large factor. A population dominated by mini-quasars could still partially ionize the IGM at $z>6$, but its contribution could be severely constrained if the X-ray background is further resolved into discrete sources.

Finally, Hansen \& Haiman (2004) proposed reionization by decaying sterile neutrinos at high-redshift. However, Mapelli \& Ferrara (2005) showed that by satisfying limits on the X-ray, optical and near-IR cosmic background, the total Thomson optical depth from sterile neutrinos is small, and they could have only played a minor role in reionization. Chen \& Kaminkowski (2003) and Pierpaoli (2003) show that the number of decays needed to reionize the universe at $z \sim 20$ will produce a CMB inconsistent with observations.

Furlanetto et al. (2004b) show that low frequency observations of the power spectrum of brightness temperature fluctuations in the $\mathrm{HI} 21 \mathrm{~cm}$ line from the neutral IGM during reionization will be able to differentiate between uniform versus HII region dominated reionization.

\section{HI $21 \mathrm{~cm}$ probes of Reionization}

The $21 \mathrm{~cm}$ line of neutral hydrogren presents a unique probe of the evolution of the neutral intergalactic medium (IGM), and cosmic reionization. Furlanetto \& Briggs (2004) point out some of the advantages of using the HI line in this regard: (i) unlike $\operatorname{Ly} \alpha$ (ie. the Gunn-Peterson effect), the $21 \mathrm{~cm}$ line does not saturate, and the IGM remains 'translucent' at large neutral fractions (Carilli et al. 2002). And (ii) unlike CMB polarization studies, the HI line provides full three dimensional (3D) information on the evolution of cosmic structure, and the technique involves imaging the neutral IGM directly, and hence can easily 
distiguish between different reionization models (Furlanetto et al. 2004b). HI $21 \mathrm{~cm}$ observations can be used to study the evolution of cosmic structure from the linear regime at high redshift (ie. density-only evolution), through the nonlinear, 'messy astrophysics' regime associated with luminous source formation. As such, HI measurements are sensitive to structures ranging from very large scales down to the source scale set by the cosmological Jeans mass, thereby making $21 \mathrm{~cm}$ the "richest of all cosmological data sets" (Barkana \& Loeb 2005b).

Many programs have been initiated to study the HI $21 \mathrm{~cm}$ signal from cosmic reionization, and beyond. The largest near-term efforts are the Mileura Wide Field Array $\left(\mathrm{MWA}^{2}\right)$, the Primeval Structure Telescope $\left(\mathrm{PAST}^{3}\right)$, and the Low Frequency Array $\left(\mathrm{LOFAR}^{4}\right)$. These telescopes are being optimized to study the power spectrum of the HI $21 \mathrm{~cm}$ fluctuations. The VLA-VHF ${ }^{5}$ system is designed specifically to set limits on the cosmic Strömgren spheres around $z \sim 6$ to 6.4 SDSS QSOs. In the long term the Square Kilometer Array $\left(\mathrm{SKA}^{6}\right)$ should have the sensitivity to perform true three dimensional imaging of the neutral IGM in the $21 \mathrm{~cm}$ line during reionization. And at the lowest frequencies $(<80 \mathrm{MHz})$, the Long Wavelength Array $\left(\mathrm{LWA}^{7}\right)$, and eventually the Lunar array (LUDAR; Corbin et al 2005; Maccone 2005), are being designed for the higher $z \mathrm{HI} 21 \mathrm{~cm}$ signal from the PGM.

In this section we summarize the current theories and capabilities for detecting the neutral IGM during, and prior to, reionization using the $\mathrm{HI} 21 \mathrm{~cm}$ line (see

\footnotetext{
${ }^{2}$ web.haystack.mit.edu/arrays/MWA/LFD/index.html

${ }^{3}$ web.phys.cmu.edu/past/

${ }^{4}$ www.lofar.org/

${ }^{5}$ cfa-www.harvard.edu/dawn/

${ }^{6}$ www.skatelescope.org/

${ }^{7}$ lwa.unm.edu/index.shtml
} 
also Carilli 2005; Furlanetto, Oh, \& Briggs 2006).

\subsection{The physics of the neutral IGM}

The physics of radiative transfer of the HI $21 \mathrm{~cm}$ line through the neutral IGM have been considered in detail by many authors (Scott \& Rees 1990; Bharadwaj \& Ali 2005; Furlanetto \& Briggs 2004; Zaldariagga et al. 2004b; Santos et al. 2005; Tozzi et al. 1999; Madau et al. 1997; Morales 2005). We review only the basic results here.

In analogy to the Gunn-Peterson effect for Ly $\alpha$ absorption by the neutral IGM, the optical depth, $\tau$, of the neutral hydrogen to $21 \mathrm{~cm}$ absorption for our adopted values of the cosmological parameters is:

$$
\tau=\frac{3 c^{3} \hbar A_{10} n_{H I}}{16 k_{B} \nu_{21}^{2} T_{S} H(z)} \sim 0.0074 \frac{x_{H I}}{T_{S}}(1+\delta)(1+z)^{3 / 2}\left[H(z) /\left(\frac{d v}{d r}\right)\right]
$$

where $A$ is the Einstein coefficient and $\nu_{21}=1420.40575 \mathrm{MHz}$ (eg. Santos et al. 2005). This equation shows immediately the rich physics involved in studying the HI $21 \mathrm{~cm}$ line during reionization, with $\tau$ depending on the evolution of cosmic over-densities, $\delta$ (predominantly in the linear regime), the neutral fraction, $x_{H I}$ (ie. reionization), the $\mathrm{HI}$ excitation, or spin, temperature, $T_{S}$, and the velocity structure, $\frac{d v}{d r}$, including the Hubble flow and peculiar velocities.

In the Raleigh-Jeans limit, the observed brightness temperature (relative to the $\mathrm{CMB})$ due to the HI $21 \mathrm{~cm}$ line at a frequency $\nu=\nu_{21} /(1+z)$, is given by:

$$
T_{B} \approx \frac{T_{S}-T_{\mathrm{CMB}}}{1+z} \tau \approx 7(1+\delta) x_{H I}\left(1-\frac{T_{C M B}}{T_{S}}\right)(1+z)^{1 / 2} \mathrm{mK}
$$

The conversion factor from brightness temperature to specific intensity, $I_{\nu}$, is

given by: $I_{\nu}=\frac{2 k_{B}}{\left(\lambda_{21}(1+z)\right)^{2}} T_{B}=22(1+z)^{-2} T_{B} \mathrm{Jy} \mathrm{deg}^{-2}$. Equation 6 shows that for $T_{S} \sim T_{C M B}$ one expects no $21 \mathrm{~cm}$ signal. When $T_{S}>>T_{C M B}$, the brightness 
temperature becomes independent of spin temperature. When $T_{S}<<T_{C M B}$, we expect a strong negative (ie. absorption) signal against the CMB.

Tozzi et al. (1999) point out that the HI excitation temperature will equilibrate with the CMB on a timescale $\sim \frac{3 \times 10^{5}}{(1+z)}$ year, in absence of other effects. However, collisions, and resonant scattering of Ly $\alpha$ photons, can drive $T_{S}$ to the gas kinetic temperature, $T_{K}$ (Wouthuysen 1952; Field 1959; Hirata 2005). Zygelman (2005) calculates that, for the mean IGM density, collisional coupling between $T_{S}$ and $T_{K}$ becomes significant for $z \geq 30$. Madau et al. (1997) show that resonant scattering of Ly $\alpha$ photons will couple $T_{S}$ and $T_{K}$ when $J_{\alpha}>9 \times 10^{-23}(1+z)$ erg $\mathrm{cm}^{-2} \mathrm{~s}^{-1} \mathrm{~Hz}^{-1} \mathrm{sr}^{-1}$, or about one Ly $\alpha$ photon per every two baryons at $z=8$.

The interplay between the CMB temperature, the kinetic temperature, and the spin temperature, coupled with radiative transfer, lead to a number of interesting physical regimes for the HI 21cm signal (Ali 2005; Barkana \& Loeb 2004; Carilli 2005): (I) At $z>200$ equilibrium between $T_{C M B}, T_{K}$, and $\mathrm{T}_{S}$ is maintained by Thomson scattering off residual free electrons and gas collisions. In this case $T_{S}=T_{C M B}$ and there is no $21 \mathrm{~cm}$ signal. (II) At $z \sim 30$ to 200 , the gas cools adiabatically, with temperature falling as $(1+z)^{2}$, ie. faster than the $(1+z)$ for the CMB. However, the mean density is still high enough to couple $T_{S}$ and $T_{K}$, and the HI 21cm signal would be seen in absorption against the CMB (Sethi 2005). (III) At $z \sim 20$ to 30 , collisions can no longer couple $T_{K}$ to $T_{S}$, and $T_{S}$ again approaches $T_{C M B}$. However, the Ly $\alpha$ photons from the first luminous objects (Pop III stars or mini-quasars), may induce local coupling of $T_{K}$ and $T_{S}$, thereby leading to some $21 \mathrm{~cm}$ absorption regions (Cen 2006). At the same time, Xrays from these same objects could lead to local IGM warming above $T_{C M B}$ (Chen et al. 2003). Hence one might expect a patch-work of regions with no signal, 
absorption, and perhaps emission, in the $21 \mathrm{~cm}$ line. (IV) At $z \sim 6$ to 20 all the physical processes come to play. The IGM is being warmed by hard Xrays from the first galaxies and black holes (Loeb \& Zaldarriaga 2004; Barkana \& Loeb 2004; Ciardi \& Madau 2003), as well as by weak shocks associated with structure formation (Furlanetto, Zaldarriaga, \& Hernquist 2005; Wang \& Hu 2005), such that $T_{K}$ is likely larger than $T_{C M B}$ globally (Furlanetto et al. 2004b). Likewise, these objects are reionizing the universe, leading to a fundamental topological change in the IGM, from the linear evolution of large scale structure, to a bubble dominated era of HII regions (Furlanetto et al. 2005a).

\subsection{Capabilities to detect HI $21 \mathrm{~cm}$ signals from reionization}

The HI 21cm signature of the neutral IGM during, and prior to, reionization can be predicted analytically, using a standard Press-Schechter type of analysis of linear structure formation, plus some recipes to approximate non-linear evolution (Santos et al. 2005; Zaldariagga et al. 2004a; Ali 2005; Bharadwaj \& Ali 2005; Wang \& Hu 2005; Gnedin \& Shaver 2004), or through the use of numerical simulations (Ciardi \& Madau 2003; Fulanetto, et al. 2004b; Wang et al. 2005).

Tomography: Figure 13 shows the expected evolution of the HI $21 \mathrm{~cm}$ signal during reionization based on numerical simulations (Zaldaraiagga et al. 2004a; Furlanetto et al. 2004a; Furlanetto et al. 2004b). They find that the mean HI signal is about $T_{B} \sim 25 \mathrm{mK}$ prior to reionization, with fluctuations of a few $\mathrm{mK}$ on arcmin scales due to linear density evolution. In this simulation, the HII regions caused by galaxy formation are seen in the redshift range $z \sim 8$ to 10 ,

reaching scales up to $2^{\prime}$ (frequency widths $\sim 0.3 \mathrm{MHz} \sim 0.5 \mathrm{Mpc}$ physical size). These regions have (negative) brightness temperatures up to $20 \mathrm{mK}$ relative to 
the mean HI signal. This corresponds to $5 \mu \mathrm{Jy} \mathrm{beam}^{-1}$ in a $2^{\prime}$ beam at $140 \mathrm{MHz}$.

The point source rms sensitivity (dual polarization) in an image from a synthesis radio telescope is given by:

$$
\mathrm{rms}=\left(\frac{1.9}{\left(\Delta \nu_{k H z} t_{h r}\right)^{0.5}}\right)\left(\frac{T_{\text {sys }}}{\epsilon_{e f f} A_{\text {ant }} N_{\text {ant }}}\right) \quad \mathrm{Jy}_{\text {beam }^{-1}}
$$

where $\Delta \nu$ is the channel width in $\mathrm{kHz}, t$ is the integration time in hours, $A_{\text {ant }}$ is the collecting area of each element in the array $\left(\mathrm{m}^{2}\right), \epsilon_{e f f}$ is the aperture efficiency, and $N$ is the number of elements $(\epsilon A N=$ total effective collecting area of the array). At low frequency, the system temperature, $T_{\text {sys }}$, is dominated by the synchrotron foreground, and behaves as: $T_{F G} \sim 100\left(\frac{\nu}{200 \mathrm{MHz}}\right)^{-2.8}$, in the coldest regions of the sky. Consider the SKA, with an effective collecting area of one square kilometer at $140 \mathrm{MHz}$, distributed over $4 \mathrm{~km}, T_{\text {sys }}=300 \mathrm{~K}$, a channel width of $0.3 \mathrm{MHz}$, and integrating for one month. The rms sensitivity is then $1.3 \mu \mathrm{Jy}_{\text {beam }^{-1}}$, with a beam FWHM $\sim 2^{\prime}$. This square kilometer array will be adequate to perform three dimensional imaging of the average structure of the IGM during reionization.

Unfortunately, the nearer term low frequency path finder arrays will have $\leq$ $10 \%$ the collecting of the SKA, and will likely not be able to perform such direct 3D imaging (see Table 1 in Carilli 2005). However, these near term experiments should have enough sensitivity to probe the neutral IGM in other ways. Figure 14 shows three possible HI $21 \mathrm{~cm}$ line signatures that might be observed prior to the SKA.

Global signal: The left panel in Figure 14 shows the latest predictions of the global (all sky) increase in the background temperature due to the $\mathrm{HI} 21 \mathrm{~cm}$ line from the neutral IGM (Gnedin \& Shaver 2003). The predicted HI emission signal peaks at roughly $20 \mathrm{mK}$ above the foreground at $z \sim 10$. At higher redshift, prior 
to IGM warming, but allowing for Ly $\alpha$ emission from the first luminous objects, the HI is seen in absorption against the CMB. Since this is an all sky signal, the sensitivity of the experiment is independent of telescope collecting area, and the experiment can be done using small area telescopes at low frequency, with well controlled frequency response (Subrahmanyan, Chippendale, Ekers 2005). Note that the line signal is only $\sim 10^{-4}$ that of the mean foreground continuum emission at $\sim 150 \mathrm{MHz}$.

Power spectra: The middle panel in Figure 14 shows the predicted power spectrum of spatial fluctuations in the sky brightness temperature due to the $\mathrm{HI}$ 21cm line (Mcquinn et al. 2006). For power spectral analyses the sensitivity is greatly enhanced relative to direct imaging due to the fact that the universe is isotropic, and hence one can average the measurements in annuli in the Fourier (uv) domain, ie. the statistics of fluctuations along an annulus in the uv-plane are equivalent (Santos et al. 2005; Zaldariagga et al. 2004a; Bharadwaj \& Ali 2005; Morales 2005; Subrahmanyan et al. 1998; Bowman et al. 2005). Moreover, unlike the CMB, HI line studies provide spatial and redshift information, and hence the power spectral analysis can be performed in three dimensions.

Figure 14 includes the power spectrum arising from linear density fluctuations following the dark matter, plus the effect of reionization. The signature of reionization can be seen as a bump in the power spectrum above the density-only curve due to the formation of HII regions. The rms fluctuations at $z=10$ peak at about $10 \mathrm{mK}$ rms on scales $\ell \sim 5000 .^{8}$ A factor 2 to 4 increase in the rms might be expected over this naive (Poisson) calculation due to gas peculiar velocities, ie. infall into superclusters and along filaments (Barkana \& Loeb 2005a, Ali

\footnotetext{
${ }^{8}$ Or $\theta \sim \frac{180^{\circ}}{\ell}=2.2^{\prime}$, or $\mathrm{k} \sim \frac{\ell}{10^{4}} \mathrm{Mpc}^{-1}=0.5 \mathrm{Mpc}^{-1}$ comoving.
} 
2005; but cf. Kuhlen et al. 2005), and possible clustering of luminous sources, ie. biased galaxy formation (Furlanetto et al. 2005a; Santos et al. 2005).

Also included in Figure 14 are the noise power spectra for near and longer term radio arrays. The noise power spectrum, in $\mathrm{mK}^{2}$, in standard spherical harmonic units, $\frac{\ell(\ell+1)}{2 \pi} C_{\ell}^{N}$, for an array with (roughly) uniform coverage of the uv plane, is given by:

$$
C_{\ell}^{N}=\frac{T_{s y s}^{2}(2 \pi)^{3}}{\Delta \nu t f_{c}^{2} \ell_{\max }^{2}}
$$

where $\Delta \nu$ is the channel width in $\mathrm{Hz}, t$ is the integration time in seconds, $f_{c}$ $=$ areal covering factor of the array $=N_{\text {ant }} \frac{A_{\text {ant }}}{A_{\text {tot }}}, A_{\text {tot }}$ is the total area of the array defined by the longest baseline $\left.\left(A_{t o t} 2\right)^{2}\right)$, and $\ell_{\max }$ is set by the longest baseline $\left(b_{\max }\right)$ in the array: $\ell_{\max }=2 \pi b_{\max } / \lambda$ (Santos et al. 2005 ; for a three dimensional generalization, see Morales 2005). For example, the SKA will have $30 \%$ of its collecting area inside $1 \mathrm{~km}$, or $f_{c}=0.3$ out to $1 \mathrm{~km}$. Typical noise values for near-term arrays are $\sim 1$ to $10 \mathrm{mK} \mathrm{rms}$ in the range $\ell=10^{3}$ to $10^{4}$, for long integrations (see Figure 14). This sensitivity is adequate to constrain the power spectrum of the $\mathrm{HI} 21 \mathrm{~cm}$ fluctuations, even if true images of the emission cannot be constructed.

A point of debate has been the fraction of $\mathrm{HI}$ in collapsed objects, as opposed to the diffuse IGM (Iliev et al. 2003). This fraction has a complex dependence on structure formation history. Oh \& Mack (2003) and Gnedin (2004) conclude that the majority of the $\mathrm{HI}$ during reionization will remain in the diffuse phase, while Ahn et al. (2005) argue that most of the HI will reside in mini-halos. Iliev et al (2003), and Ahn (2005) have considered the HI $21 \mathrm{~cm}$ power spectrum due to clustering of such minihalos at high redshift. For a beam size of $1^{\prime}$, and channel width of $0.2 \mathrm{MHz}$, they predict $3 \sigma$ brightness temperature fluctuations due to 
clustered minihalos $\sim 7 \mathrm{mK}$ at $z=8.5$, decreasing to $2 \mathrm{mK}$ at $z=20$.

Absorption toward discrete radio sources: A interesting alternative to emission studies is the possibility of studying smaller scale structure in the neutral IGM by looking for HI 21cm absorption toward the first radio-loud objects (AGN, star forming galaxies, GRBs) (Carilli et al. 2004b). The rightpanel of Figure 14 shows the predicted HI $21 \mathrm{~cm}$ absorption signal toward a high redshift radio source due to the 'cosmic web' prior to reionization, based on numerical simulations (Carilli, Gnedin, Owen 2002). For a source at $z=10$, these simulations predict an average optical depth due to $21 \mathrm{~cm}$ absorption of about $1 \%$, corresponding to the 'radio Gunn-Peterson effect', and about five narrow (few km/s) absorption lines per $\mathrm{MHz}$ with optical depths of a few to $10 \%$. These latter lines are equivalent to the Ly $\alpha$ forest seen after reionization, and correspond to over-densities evolving in the linear regime $(\delta \leq 10)$. Fulanetto \& Loeb (2002), and Oh \& Mack (2003) predict a similar HI $21 \mathrm{~cm}$ absorption line density due to gas in minihalos as that expected for the $21 \mathrm{~cm}$ forest.

Fundamental to absorption studies is the existence of radio loud sources during the EoR. This question has been considered in detail by Carilli et al. (2002), Haiman et al. (2004), and Jarvis \& Rawlings (2005). They show that current models of radio-loud AGN evolution predict between 0.05 and 1 radio sources per square degree at $z>6$ with $\mathrm{S}_{150 \mathrm{MHz}} \geq 6 \mathrm{mJy}$, adequate for EoR HI $21 \mathrm{~cm}$ absorption studies with the SKA.

Cosmic Strömgren spheres: While direct detection of the typical structure of HI and HII regions may be out of reach of the near-term EoR 21cm telescopes, there is a chance that even this first generation of telescopes will be able to detect the rare, very large scale HII regions associated with luminous quasars near the 
end of reionization $(\S 4)$. The expected signal is $\sim 20 \mathrm{mK} \times x_{H I}$ on a scale $\sim 10^{\prime}$ to $15^{\prime}$, with a line width of $\sim 1$ to $2 \mathrm{MHz}$ (Wyithe \& Loeb 2004b). This corresponds to $0.5 \times x_{H I}$ mJy beam ${ }^{-1}$, for a $15^{\prime}$ beam at $z \sim 6$ to 7 . Kohler et al. (2005) calculate the expected spectral dips due to large HII regions around luminous quasars $\left(>2 \times 10^{10} \mathrm{~L}_{\odot}\right)$ during the EoR. In a typical spectrum from 100 to 180 $\mathrm{MHz}$ with a $10^{\prime}$ beam they predict on average one relatively deep (-2 to $\left.-4 \mathrm{mK}\right)$ dip per LoS on this scale. Wyithe et al. (2005b) perform a similar calculation using the evolution of the bright QSO luminosity function to predict the number of HII regions around active QSOs at $z>6$. They conclude that there should be roughly one SDSS-type HII region around an active QSO (physical radius > $4 \mathrm{Mpc}$ ) per $400 \mathrm{deg}^{2}$ field per $16 \mathrm{Mhz}$ bandwidth at $z \sim 6$, and one $R \geq 2 \mathrm{Mpc}$ region at $z \sim 8$, and up to $\sim 100 \times$ more fossil HII regions due to non-active AGN, depending on the duty cycle.

Beyond reionization: Recent calculations of the brightness temperature fluctuations due to the $21 \mathrm{~cm}$ line have extended to redshifts higher than reionization, $z>20$. Barkana \& Loeb $(2004 ; 2005 a)$ predict the power spectrum of fluctuations during the era of 'Ly $\alpha$ coupling' ( $z \sim 20$ to 30; see also Kuhlen et al. 2005 and Cen 2006). Brightness temperature fluctuations can be due to emission from clustered minihalos, plus enhanced by absorption against the CMB by the diffuse IGM, with a predicted rms $\sim 10 \mathrm{mK}$ for $\ell \sim 10^{5}$, due to a combination of linear density fluctuations, plus Poisson ('shot') noise and biasing in the Ly $\alpha$ source (ie. galaxy) distribution.

Loeb \& Zaldarriaga (2004) go even further in redshift, to $z>50$ to 200. In this regime the HI generally follows linear density fluctuations, and hence the experiments are as clean as CMB studies, and $T_{K}<T_{C M B}$, so a relatively strong 
absorption signal might be expected. They also point out that Silk damping, or photon diffusion, erases structures on scales $\ell>2000$ in the CMB at recombination, corresponding to comoving scales $=22 \mathrm{Mpc}$. The HI $21 \mathrm{~cm}$ measurements can explore this physical regime at $z \sim 50$ to 300 . The predicted rms fluctuations are 1 to $10 \mathrm{mK}$ on scales $\ell=10^{3}$ to $10^{6}\left(0.2^{o}\right.$ to $\left.1^{\prime \prime}\right)$. These observations could provide the best tests of non-Gaussianity of density fluctuations, and constrain the running power law index of mass fluctutions to large $\ell$, providing important tests of inflationary structure formation. Sethi (2005) also suggests that a large global signal, up to $-0.05 \mathrm{~K}$, might be expected for this redshift range.

Unfortunately, the sky background is very hot $>10^{4} \mathrm{~K}$ at these low frequencies $(<50 \mathrm{MHz})$, and the predicted signals are orders of magnitude below the expected sensitivities of even the biggest planned low frequency arrays in the coming decade.

\subsection{Observational challenges}

Foregrounds: The HI $21 \mathrm{~cm}$ signal from reionization must be detected on top of a much larger synchrotron signal from foreground emission $(\S 9.2)$. This foreground includes discrete radio galaxies, and large scale emission from our own Galaxy, with relative contributions of about $10 \%$ and $90 \%$, respectively. The expected HI $21 \mathrm{~cm}$ signal is about $10^{-4}$ of the foreground emission at $140 \mathrm{MHz}$.

di Matteo et al. (2002) show that, even if point sources can be removed to the level of $1 \mu \mathrm{Jy}$, the rms fluctutions on spatial scales $\leq 10^{\prime}(\ell \geq 1000)$ due to residual radio point sources will be $\geq 10 \mathrm{mK}$ just due to Poisson noise, increasing by a factor 100 if the sources are strongly clustered (see also di Matteo, Ciardi, \& Miniati 2004; Oh \& Mack 2003). 
A key point is that the foreground emission should be smooth in frequency, predominantly the sum of power-law, or perhaps gently curving, non-thermal spectra. A number of complimentary approaches have been presented for foreground removal (Morales, Bowman, \& Hewitt 2005). Gnedin \& Shaver (2003) and Wang et al (2005) consider fitting smooth spectral models (power laws or low order polynomials in log space) to the observed visibilities or images. Morales \& Hewitt (2003) and Morales (2004) present a 3D Fourier analysis of the measured visibilities, where the third dimension is frequency. The different symmetries in this $3 \mathrm{D}$ space for the signal arising from the noise-like HI emission, versus the smooth (in frequency) foreground emission, can be a powerful means of differentiating between foreground emission and the EoR line signal. Santos et al. (2005), Bharadwaj \& Ali (2005), Zaldariagga et al. (2004a) perform a similar analysis, only in the complementary Fourier space, meaning cross correlation of spectral channels. They show that the $21 \mathrm{~cm}$ signal will effectively decorrelate for channel separations $>1 \mathrm{MHz}$, while the foregrounds do not. The overall conclusion of these methods is that spectral decomposition should be adequate to separate synchrotron foregrounds from the HI $21 \mathrm{~cm}$ signal from reionization at the $\mathrm{mK}$ level.

Ionosphere: A second potential challenge to low frequency imaging over wide fields is phase fluctuations caused by the ionosphere. These fluctuations are due to index of refraction fluctuations in the ionized plasma, and behave as $\Delta \phi \propto \nu^{-2}$. Morever, the typical 'isoplanatic patch', or angle over which a single phase error applies, is a few to 10 degrees (physical scales of 10's $\mathrm{km}$ in the ionosphere), depending on frequency (Cotton et al. 2004; Lane et al. 2004). Fields larger than the isoplanatic patch will have multiple phase errors across the field, and hence 
cannot be corrected through standard (ie. single solution) phase self-calibration techniques. New wide field self-calibration techniques, involving multiple phase solutions over the field, or a 'rubber screen' phase model (Cotton et al. 2004; Hopkins et al. 2003), are being developed that should allow for self-calibration over wide fields.

Interference: Perhaps the most difficult problem facing low frequency radio astronomy is terrestrial (man-made) interference (RFI). The relevant frequency range corresponds to 7 to $200 \mathrm{MHz}(z=200$ to 6$)$. These are not protected frequency bands, and commercial allocations include everything from broadcast radio and television, to fixed and mobile communications.

Many groups are pursuing methods for RFI mitigation and excision (see Ellingson 2004). These include: (i) using a reference horn, or one beam of a phased array, for constant monitoring of known, strong, RFI signals, (ii) conversely, arranging interferometric phases to produce a null at the position of the RFI source, and (iii) real-time RFI excision using advanced filtering techniques in time and frequency, of digitized signals both pre- and post-correlation. The latter requires very high dynamic range (many bit sampling), and very high frequency and time resolution.

In the end, the most effective means of reducing interference is to go to the remotest sites. The MWA and PAST have selected sites in remote regions of Western Australia, and China, respectively, because of known low RFI environments. Of course, the ultimate location would be the far-side of the moon.

The technical challenges to HI $21 \mathrm{~cm}$ observations of reionization are many. Use of spectral decomposition to remove the foregrounds requires careful control of the synthesize beam as a function of frequency, with the optimal solution being 
a telescope design where the synthesized beam is invariant as a function of frequency (Subrahmanyan et al. 2005). High dynamic range front ends are required to avoid saturation in cases of strong interference, while fine spectral sampling is required to avoid Gibbs ringing in the spectral response. The polarization response must be stable and well calibrated to remove polarized foregrounds (Haverkorn et al. 2004). Calibation in the presence of a structured ionospheric phase screen requires new wide field calibration techniques. The very high data rate expected for many element $\left(\geq 10^{3}\right)$ arrays requires new methods for data transmission, cross correlation, and storage. At the lowest frequencies, $\leq 20 \mathrm{MHz}$ or so, where we hope to study the PGM, phase fluctuations and the opacity of the ionosphere becomes problematic, leading to the proposed LUDAR project on the far side of the moon. The far side of the moon is also the best location in order to completely avoid terrestrial interference.

\section{Summary}

The few years since the seminal theoretical reviews of Barkana \& Loeb have seen dramatic progress in determining observational constraints on cosmic reionization. Figure 15 shows the current limits on the cosmic neutral fraction versus redshift. The observations paint an interesting picture. On the one hand, studies of GP optical depths and variations, and the GP 'gap' distribution, as well as of the thermal state of the IGM at high $z$, and of cosmic Strömgren spheres and surfaces around the highest redshift QSOs, suggest a qualitative change in the state of the IGM at $z \sim 6$. These data indicate a significant neutral fraction,

$x_{H I}>10^{-3}$, and perhaps as high as 0.1 , at $z \geq 6$, as compared to $x_{H I} \leq 10^{-4}$ at $z<5.5$. The IGM characteristics at this epoch are consistent with the end of 
the 'percolation' stage of reionization (Gnedin \& Fan 2006). On the other hand, transmission spikes in the GP trough and study of the evolution of Ly $\alpha$ galaxy luminosity function indicate a neutral fraction smaller than $50 \%$ at $z \sim 6.5$. Moreover, the measurement of the large scale polarization of the CMB implies a significant ionization fraction extending to higher redshifts, $z \sim 15$ to 20 . Moreover, the measurement of the large scale polarization of the CMB suggests a significant ionization fraction extending to higher redshifts, $z \sim 11 \pm 3$.

We emphasize that all these measurements have implicit assumptions and uncertainties, as discussed throughout. Indeed, the GP effect and CMB large scale polarization studies can be considered complimentary probes of reionization, with optical depth effects limiting GP studies to the end of reionization, while CMB studies are weighted toward the higher redshifts, when the densities were higher. The data argue against a simple reionization history in which the IGM remains largely neutral from $z \sim 1100$ to $z \sim 6-7$, with a single phase transition at $z \sim 6$ (the "late" model in Figure 15), as well as against a model in which the Universe reaches complete ionization at $z \sim 15-20$ and remained so ever since (the "early" model in Figure 15). These facts, combined with the large line of sight variations at the end of reionization as indicated by Gunn-Peterson measurements, suggest a more extended reionization history. Interestingly, the latest theoretical models with reionization caused by Population II star formation are consistent with both GP optical depth and WMAP CMB polarization measurement (e.g. Gnedin \& Fan 2006). Current data do not present strong evidence for a dominant contribution by metal-free Population III star formation at $z>15$ to reionization (Haiman \& Bryan 2006), and have weak constraints on, although do not require, models with multiple episodes of reionization (the "double" model 
in Figure 15), which were suggested by a possible high CMB Thomspon optical depth based on early WMAP measurements.

Overall, we agree with the assessment of Furlanetto et al. (2004b) that reionization is less an event than a process, extended in both time and space. Much emphasis has been given to the determination of the 'reionization redshift'. However, current observations suggest that, unlike recombination, which occurs over a very narrow range in fractional redshift, $z_{\text {recomb }}=1089 \pm 1$, cosmic reionization occurs over a fairly large fractional redshift, from $z \sim 6$ up to as high as $z \sim 14$. The redshift of HII region overlap might be quite different than the redshift at which the IGM is largely $(>50 \%)$ ionized. It may also differ in different parts of the universe. The implication is that the formation of the first liminous objects (stars and AGN) occurs in a 'twilight zone', heavily obscured at (observed) optical wavelengths by a partially neutral IGM, and observable only at near-IR through radio wavelengths, and in the hard Xrays.

We also consider the sources responsible for reionization. Current data are consistent with star forming galaxies, in particular, relatively low luminosity galaxies, as being the dominant sources of reionizing photons. However, again, there are a number of important, and poorly constrained, parameters in these calculations, including the IMF and the UV escape fraction, and we certainly cannot rule-out a significant contribution from mini-QSOs.

Lastly, we show that low frequency radio telescopes currently under construction should be able to make the first direct measurements of $\mathrm{HI} 21 \mathrm{~cm}$ emission from (and/or absorption by) the neutral IGM. These observations will present a clear picture of the reionization process, and the evolution of the neutral IGM into the dark ages. 
Acknowledgements: CC thanks the Max-Planck-Gesellschaft and the HumboldtStiftung for support through the Max-Planck-Forschungspreis, K. Menten and the MPIfR for their hospitality, and S. Furlanetto for extensive comments. XF thanks supports from NSF grant AST 03-07384, a Sloan Research Fellowship and Packard Fellowship for Science and Engineering. BK thanks Nathan Miller for his contributions to this review and Steven Furlanetto, Manoj Kaplinghat, and Meir Shimon for helpful comments on this manuscript. CMBFAST was used in the preparation of this review. The National Radio Astronomy Observatory (NRAO) is a facility of the National Science Foundation, operated under cooperative agreement by Associated Universities, Inc.

\section{Literature Cited}

Anderson, Hogan, Williams, \& Carswell(1999)Anderson, S. F., Hogan, C. J., Williams, B. F., \& Carswell, R. F. 1999, A.J., 117: 56-62

Ahn et al. 2005Ahn, K., Shapiro, P., Alvarez, M., Iliev, T., Martel, H., Ryu, D. 2005, New AR, in press (astroph/0509651)

Ajiki et al. (2003)Ajiki, M., et al. 2003, A.J., 126: 2091-2107

Ali 2005Ali, Sk. 2005, MNRAS, in press, (astroph-0503237)

Bahcall \& Salpeter (1965)Bahcall, J. N., \& Salpeter, E. E. 1965, Ap.J., 142: $1677-1680$

Barkana \& Loeb 2001Barkana, R., Loeb, A. 2001, Phys. Rep. 349, 125-238

Barkana \& Loeb (2004)Barkana, R., \& Loeb, A. 2004, Ap.J., 601, 64-69

Barkana \& Loeb 2005aBarkana, R., Loeb, A. 2005a, ApJ, 626, 1-11

Barkana \& Loeb 2005bBarkana, R., Loeb, A. 2005b, ApJ, 624, L65-68

Barkana \& Loeb 2005cBarkana, R., Loeb, A. 2005c, MNRAS, 363, L36 
Barkats et al.(2005)Barkats, D., et al. 2005, Ap.J., 619, L127-130

Barton et al. (2004)Barton, E. J., Davé, R., Smith, J.-D. T., Papovich, C., Hernquist, L., \& Springel, V. 2004, Ap.J. Lett., 604: L1-L4

Becker et al. (2006)Becker06] Becker, G. D., Sargent, W. L. W., Rauch. M., \& Simcoe, R. A. 2006, ApJ, in press (astro-ph/0510747)

Becker et al. (2001)Becker, R. H., et al. 2001, A.J., 122: 2850-2857

Bertoldi et al. 2003Bertoldi, F., Carilli, C., Cox, P. et al. 2003, A\& A, 406, L55-58

Bertoldi et al. 2004Bertoldi, F., Cox, P., Neri, R. et al. 2004, A\& A, 409, L47-50

Bharadwaj \& Ali 2005Bharadwaj, S. \& Ali, Sk. 2005, MNRAS, 356, 1519-1428

Bi 1993Bi, H. 1993, Ap.J., 405: 479-490

Bouwens et al.(2004)Bouwens, R. J., et al. 2004a, Ap.J. Lett., 606, L25-28

Bouwens et al. 2004Bouwens, R.J, et al. 2004c, Ap.J. Lett., 616, L79-82

Bouwens et al. 2005Bouwens, R.J, Illingworth, G.D., Thompson, R.I., \& Franx, M. 2005, Ap.J., 624, L5-8

Bouwens et al. 2006Bouwens, R. J., Illingworh, G. D., Blakeslee, J. P., \& Franx, M. 2006, ApJ, in press (astro-ph/0509641)

Bowman et al. 2005Bowman, J, Morales, M., Hewitt, J. 2005, ApJ, 638, 20-28

Bunker et al. 2004Bunker, A. J., Stanway, E. R., Ellis, R. S., \& McMahon, R. G. 2004, MNRAS, 355: 374-384

Carilli et al 2004aCarilli, C., Gnedin, N., Furlanetto, S., \& Owen, F. 2004, NewAR, 48, 1053-1061

Carilli et al. 2004bCarilli, C., Furlanetto, S., Briggs, F., Jarvis, M., Rawlings, S., Falcke, H. 2004, NewAR, 48, 1029-1038

Carilli et al. 2002Carilli, C., Gnedin, N., Owen, F. 2002, ApJ, 577, 22-30

Cen 2003aCen, R. 2003a, Ap.J., 591, 12-37 
Cen 2003bCen, R. 2003b, Ap.J., 591, L5-8

Cen 2005Cen, R. 2005, ApJL, submitted (astro-ph/0507014)

Cen 2006Cen, R. 2006, ApJL, submitted (astro-ph/0601010)

Cen \& Haiman 2000Cen, R., \& Haiman, Z. 2000, Ap.J. Lett., 542: L75-L78

Cen, Haiman, \& Mesinger 2005Cen, R., Haiman, Z., \& Mesinger, A. 2005, Ap.J., 621: $89-94$

Cen \& McDonald 2002Cen, R., \& McDonald, P. 2002, Ap.J., 570: 457-462

Cen, Miralda-Escude, Ostriker, \& Rauch 1994Cen, R., Miralda-Escude, J., Ostriker, J. P., \& Rauch, M. 1994, Ap.J. Lett., 437: L9-L12

chary05Chary, R., Stern, D., Eisenhardt, P. 2005, ApJ, 635, L5

Chen et al. 2005Chen, H., Prochaska, J. X., Bloom, J. S., Thompson, I. B, 2005, ApJ, 634, L25

Chen \& Kamionkowski(2004)Chen, X., \& Kamionkowski, M. 2004, Phys. Rev. D., 70,043502

Chen \& Miralda-Escude 2004Chen, X. \& Miralda-Escude, J. 2004, ApJ, 602, 1-11

Chiu et al. 2003Chiu, W. A., Fan, X., \& Ostriker, J. P. 2003, ApJ, 59 9. 759

Ciardi \& Ferrara 2005Ciardi, B., \& Ferrara, A. 2005, Space Science Reviews, 116 : $625-705$

Ciardi \& Madau 2003Ciardi, B., Madau, P. 2003, ApJ, 596, 1-8

Ciardi et al. 2003Ciardi, B., Stoehr, F., White, S. 2004, MNRAS, 343, 1101-1109

Ciardi et al. 2001Ciardi, B., Ferrara, A., Marri, S., \& Raimondo, G. 2001, MNRAS, 324: 381-388

Ciardi \& Loeb 2000Ciardi, B., \& Loeb, A. 2000, Ap.J., 540: 687-696

Colombo et al.(2005)Colombo, L. P. L., Bernardi, G., Casarini, L., Mainini, R., Bonometto, S. A., Carretti, E., \& Fabbri, R. 2005, Astron. and Astrophys., 
435,413

Cool et al. (2006)Cool06] Cool, R. J., et al. 2006, AJ, in press

Corbin et al. 2005Corbin, M. et al. 2005, exploratory proposal to NASA

Cotton et al. 2004Cotton, W., Condon, J., Perley, R. et al. 2004, SPIE, 5489, $180-189$

Davidsen, Kriss, \& Wei(1996)Davidsen, A. F., Kriss, G. A., \& Wei, Z. 1996, Nature, 380: 47-49

Dawson et al.(2002)Dawson, K. S., Holzapfel, W. L., Carlstrom, J. E., Joy, M., LaRoque, S. J., Miller, A. D., \& Nagai, D. 2002, Ap.J., 581, 86

Dijkstra, Haiman, \& Scharf 2005Dijkstra, M., Haiman, Z. \& Loeb, A. 2005, ApJ, $613,646-654$

Di Matteo et al 2002Di Matteo, T., Rosalba, P., Abel, T., Rees, M. 2002, MNRAS, 564, 576-580

di Matteo et al. 2004Di Matteo, T., Ciardi, B., \& Miniati, F. 2004, MNRAS, 355, $1053-1065$

Djorgovski, Castro, Stern, \& Mahabal 2001Djorgovski, S. G., Castro, S., Stern, D., \& Mahabal, A. A. 2001, Ap.J. Lett., 560: L5-L8

Djorgovski et al. 2005Djorgovski, S. G., Bogosavljevic, M., \& Mahabal, A. 2005, atro-ph/0509065

Dodelson(2003)Dodelson, S. 2003, Modern cosmology / Scott Dodelson. Amsterdam (Netherlands): Academic Press. ISBN 0-12-219141-2, 2003, XIII + 440 p.,

Ellingson05Ellingson, S. 2005, Exp. Astron., 17, 261-267

Eyles et al. 2005Eyles, L. et al. 2005, MNRAS, 364, 443-450

Fan et al. 2000Fan, X., et al. 2000, A.J., 120: 1167-1174 
Fan et al. 2001aFan, X., et al. 2001a, A.J., 121: 54-65

Fan et al. 2001bFan, X., et al. 2001b, A.J., 122: 2833-2849

Fan et al. 2002Fan, X., Narayanan, V. K., Strauss, M. A., White, R. L., Becker, R. H., Pentericci, L., \& Rix, H.-W. 2002, A.J., 123: 1247-1257

Fan et al. 2003Fan, X., et al. 2003, A.J., 125: 1649-1659

Fan et al. 2004Fan, X., et al. 2004, A.J., 128: 515-522

Fan et al. 2006aFan et al. 2006a, AJ, in press (astro-ph/0512080)

Fan et al. 2006bFan et al. 2006b, AJ, submitted (astro-ph/0512082)

Fernández-Soto, Lanzetta, \& Chen(2003)Fernández-Soto, A., Lanzetta, K. M., \& Chen, H.-W. 2003, MNRAS, 342: 1215-1221

Field 1959Field, G.B. 1959, ApJ, 129, 551-565

Furlanetto \& Briggs 2004Furlanetto, S. \& Briggs, F. 2004, New AR, 48, 10391052

Furlanetto, Og, Briggs 2006Furlanetto, S., Oh, S.P., Briggs, F. 2006, Physics Reports, in prep

Furlanetto, OhFurlanetto, S., Oh, S.P. 2005, MNRAS, 363, 1031 - 1048

Furlanetto \& Loeb 2002Furlanetto, S, \& Loeb, A. 2002, ApJ, 579, 1-9

Furlanetto et al. 2004aFurlanetto, S., Zaldarriaga, M. Hernquist, L. 2004, ApJ, $613,16-22$

Furlanetto et al. 2004bFurlanetto, S., Sokasian, A., Hernquist, L. 2004, MNRAS $347,187-195$

Furlanetto et al. 2005aFurlanetto, S., McQuinn, D., Hernquist, L. 2005, MNRAS, 365,115

Furlanetto et al. 2005bFurlanetto, S. R., Zaldarriaga, M., \& Hernquist, L. 2006, MNRAS, 365, 1012-1020 
Furlanetto, Hernquist, \& Zaldarriaga 2004cFurlanetto, S. R., Hernquist, L., \& Zaldarriaga, M. 2004, MNRAS, 354: 695-707

Furlanetto \& Loeb 2003Furlanetto, S. R., \& Loeb, A. 2003, Ap.J., 588: 18-34

Gallerani et al. 2006Gallerani, S., Roy Choudhury, T., Ferrara, A. 2006, MNRAS, submitted (astro-ph/0512129)

Giallongo et al. 2002Giallongo, E., Cristiani, S., D'Odorico, S., \& Fontana, A. 2002, Ap.J. Lett., 568: L9-12

Giallongo et al. 1994Giallongo, E., D’Odorico, S., Fontana, A., McMahon, R. G., Savaglio, S., Cristiani, S., Molaro, P., \& Trevese, D. 1994, Ap.J. Lett., 425: L14

Giavalisco et al. 2004Giavalisco, M., et al. 2004, Ap.J. Lett., 600: L93-98

Gnedin \& Ostriker 1997Gnedin, N. Y., \& Ostriker, J. P. 1997, Ap.J., 486: 581598

Gnedin 2000Gnedin, N. 2000, ApJ, 535, 530-554

Gnedin \& Shaver 2003Gnedin, N. \& Shaver, P. 2004, ApJ, 608, 611-621

Gnedin 2004Gnedin, N. 2004, ApJ, 610, 9-13

Gnedin \& Prada 2004Gnedin, N. Y., \& Prada, F. 2004, Ap.J. Lett., 608: L77-L80

Gnedin \& Fan 2006Gnedin, N. Y., \& Fan, X. 2006, Ap.J.submitted (astroph/0603794)

Gruzinov \& Hu (1998)Gruzinov, A., and Hu, W. 1998, Ap.J., 508, 435

Gunn \& Peterson 1965Gunn, J. E., \& Peterson, B. A. 1965, Ap.J., 142: 16331641

Haardt \& Madau 1996Haardt, F., \& Madau, P. 1996, Ap.J., 461, 20-37

Haehnelt et al. 2001Haehnelt, M. G., Madau, P., Kudritzki, R., \& Haardt, F. 2001, Ap.J. Lett., 549, L151-L154 
Haiman 2002Haiman, Z. 2002, Ap.J. Lett., 576: L1-L4

Haiman \& Cen 2005Haiman, Z., \& Cen, R. 2005, Ap.J., 623: 627-631

Haiman et al. 2004Haiman, Z., Quartaert, E., Bower, G. 2004, ApJ, 612, 698-705

Haiman \& Holder 2003Haiman, Z. \& Holder, G. P. 2003, ApJ, 595, 1

Haiman \& Knox(1999)Haiman, Z., \& Knox, L. 1999, ASP Conf. Ser. 181: Microwave Foregrounds, 181, 227

haiman \& Bryan 2006Haiman, Z. \& Bryan, G.L. 2006, ApJ letters, in press (astroph/0603541)

Haislip et al. 2005Haislip, J. et al. 2005, Nature, submitted (astro-ph/0509660)

Hansen \& Haiman 2004Hansen, S. H., \& Haiman, Z. 2004, ApJ, 600: 26-31

Haverkorn et al. 2004Haverkorn, M., Katgert, P., de Bruyn, A. 2004, A\& A, 427, $549-559$

Hernquist et al. 1996Hernquist, L., Katz, N., Weinberg, D. H., \& MiraldaEscudé, J. 1996, Ap.J. Lett., 457: L51- L54

Hopkins et al. 2003Hopkins, P., Doeleman, S., Lonsdale, C. 2003, AAS, 203, 4005

Hirata, C.M. 2005, MNRAS, in press

Holder et al.(2003)Holder, G. P., Haiman, Z., Kaplinghat, M., \& Knox, L. 2003, Ap.J., 595, 13

Horton et al. 2004Horton, A., Parry, I., Bland-Hawthorn, J., Cianci, S., King, D., McMahon, R., \& Medlen, S. 2004, SPIE, 5492: 1022-1032

Hu (2000)Hu, W. 2000, Ap.J., 529, 12

Hu \& White(1997)Hu, W., \& White, M. 1997, New Astronomy, 2, 323

Hu \& Okamoto(2002)Hu, W., \& Okamoto, T. 2002, Ap.J., 574, 566

Hu \& Holder 2003Hu, W. \& Holder, G., 2003, Phys. Rev. D, 68, 3001-3004

Hu et al. 2002Hu, E. M., Cowie, L. L., McMahon, R. G., Capak, P., Iwamuro, 
F., Kneib, J.-P., Maihara, T., \& Motohara, K. 2002, Ap.J. Lett., 568: L75-L79

Hu et al. 2004Hu, E. M., Cowie, L. L., Capak, P., McMahon, R. G., Hayashino, T., \& Komiyama, Y. 2004, A.J., 127: 563-575

Hu et al. 2005Hu, E. M., Cowie, L. L., Capak, P., Kakazu, Y.. 2005, to ppear in IAU 199 Conf. Proc.: "Probing Galaxies through Quasar Absorption Lines," eds. Williams, Shu, Menard (astro-ph/0509616)

Hui \& Haiman 2003Hui, L., \& Haiman, Z. 2003, Ap.J., 596: 9-18

Iliev et al. 2003Iliev, I., Scannapieco, E., Martel, H., Schapiro, P. 2003, MNRAS, $341,81-90$

Iliev et al. 2005Iliev, I., Mellema, G., Pen, U., Merz, H., Shapiro, P., Alvarez, M. 2006, MNRAS, in press (astroph/0512187)

Inoue et al. 2005Inoue, A. K., Iwata, I., Deharveng, J.-M., Buat, V., \& Burgarella, D. 2005, A\&A, 435: 471-482

Jakobsen et al.(1994)Jakobsen, P., Boksenberg, A., Deharveng, J. M., Greenfield, P., Jedrzejewski, R., \& Paresce, F. 1994, Nature, 370: 35-39

Jarvis \& Rawlings 2005Jarvis, M. \& Rawlings, S. 2005, New AR, 48, 1173-1185

Jarosik et al.(2003)Jarosik, N., et al. 2003, Ap.J.Supplement, 145, 413

Kashikawa et al. 2005Kashikawa, N. et al. 2005, ApJ, 637, 631

kashilnskyKashlinsky, A., Arendt, R., Mather, J., Moseley, S. 2005, Nature, 438, $45-50$

Kamionkowski et al.(1997)Kamionkowski, M., Kosowsky, A., \& Stebbins, A. 1997, Phys. Rev. D., 55, 7368

Kaplinghat et al.(2003)Kaplinghat, M., Chu, M., Haiman, Z., Holder, G. P., Knox, L., \& Skordis, C. 2003, ApJ, 583, 24

Kaplinghat, Knox and Song (2003)Kaplinghat M., Knox, L., \& Song, Y., 2003, 
Phys. Rev. Lett., 91, 241301

Kawai et al. 2006Kawai, N., et al. 2006, Nature, in press (astro-ph/0512052)

Keating et al.(1998)Keating, B., Timbie, P., Polnarev, A., \& Steinberger, J. 1998, Ap.J., 495, 580

Keating et al.(2001)Keating, B. G., O'Dell, C. W., de Oliveira-Costa, A., Klawikowski, S., Stebor, N., Piccirillo, L., Tegmark, M., \& Timbie, P. T. 2001, Ap.J., 560, L1

Keating \& Miller (2005)Keating, B. and Miller, N. 2005, astro-ph 0508269, to appear in New Astronomy Reviews

Kesden et al.(2002)Kesden, M., Cooray, A., \& Kamionkowski, M. 2002, Physical Review Letters, 89, 011304

Kennefick, Djorgovski, \& de Carvalho 1995Kennefick, J. D., Djorgovski, S. G., \& de Carvalho, R. R. 1995, A.J., 110: 2553-2565

Knox et al.(1998)Knox, L., Scoccimarro, R., \& Dodelson, S. 1998, Physical Review Letters, 81, 2004

Knox \& Song(2002)Knox, L., \& Song, Y.-S. 2002, Physical Review Letters, 89, 011303

Knox(2003)Knox, L. 2003, New Astronomy Review, 47, 883

Kodaira et al. 2003Kodaira, K., et al. 2003, PASJ, 55: L17-L21

Kogut et al.(2003)Kogut, A., et al. 2003, Ap.J.Suppl., 148, 161

Kosowsky(2003)Kosowsky, A. 2003, New Astronomy Review, 47, 939

Kohler et al. 2005Kohler, K., Gnedin, N., Miralda-Escude, J., Shaver, P. 2005, ApJ, 633, 552

Kohler et al. 2006Kohler, K., Gnedin, N., \& Hamilton, A. J. S. 2006, submitted (astro-ph/0511627) 
Kriss et al.(2001)Kriss, G. A., et al. 2001, Science, 293: 1112-1116

Kuhlen05Kuhlen, M., Madau, P., Montgomery, R. 2005, ApJ, 637, L1

Kurk et al. 2004Kurk, J. D., Cimatti, A., di Serego Alighieri, S., Vernet, J., Daddi, E., Ferrara, A., \& Ciardi, B. 2004, A\&A, 422: L13-L17

Lamarre et al.(2003)Lamarre, J. M., et al. 2003, New Astronomy Review, 47, 1017

Lane et al. 2004Lane, W., Cohen, A., Cotton, W., et al. 2004, SPIE, 5489, 354361

Lawrence et al. 2006Lawrence, A., et al. 2006, MNRAS, submitted

Leitch et al.(2005)Leitch, E. M., Kovac, J. M., Halverson, N. W., Carlstrom, J. E., Pryke, C., \& Smith, M. W. E. 2005, Ap.J., 624, 10

Lidz et al. 2002Lidz, A., Hui, L., Zaldarriaga, M., \& Scoccimarro, R. 2002, Ap.J., 579: 491-499

Loeb \& Barkana 2000Loeb, A., Barkana, R. 2001, ARAA, 39, 19-66

Loeb \& Zaldarriaga 2004Loeb, A. \& Zaldarriaga, M. 2004, Phys.Rev. Lett. 92, $1301-1304$

Maccone 2004Maccone, C. 2004, 35th COSPAR Assembly, p. 1415-1419

Madau et al. 1997Madau, P., Haardt, F., \& Rees, M. J. 1997, Ap.J., 514, 648-659

Madau et al. 2004Madau, P., Rees, M. J., Volonteri, M., Haardt, F., \& Oh, S. P. 2004, Ap.J., 604: 484-494

Madau et al. 2001Madau, P., Ferrara, A., \& Rees, M. J. 2001, Ap.J., 555: 92-105

Madau \& Rees 2000Madau, P., \& Rees, M. J. 2000, Ap.J. Lett., 542: L69-L73

Madau et al. 1997Madau, P., Meiksin, A., Rees, M. 1997, ApJ, 475, 429-444

Mahabal05Mahabal, A., Stern, D., Bogosavljevic, M., Djorgovski, S., Thompson, D. 2005, ApJ, 634, L9 
malhotraMalhotra, S. \& Rhoads, J. 2005, ApJ, 617, L5

Malhotra et al. 2005Malhotra, S., et al. 2005, Ap.J., 626: 666-679

Malhotra \& Rhoads 2004Malhotra, S., \& Rhoads, J. E. 2004, Ap.J. Lett., 617: L5-L8

Mapelli \& Ferrara 2005Mapelli, M., \& Ferrara, A. 2005, MNRAS, 364, 2

Martin \& Sawicki 2004Martin, C. L., \& Sawicki, M. 2004, Ap.J., 603: 414-424

McDonald et al. 2000McDonald, P., Miralda-Escudé, J., Rauch, M., Sargent, W. L. W., Barlow, T. A., Cen, R., \& Ostriker, J. P. 2000, Ap.J., 543: 1-23

McDonald \& Miralda-Escudé 2001McDonald, P., \& Miralda-Escudé, J. 2001, Ap.J. Lett., 549: L11-L14

mcphersonMcPherson, A., Born, A., Sutherland, W., Emerson, J. 2004, SPIE, $5489,638-649$

McQuinn et. al. (2005)McQuinn, M., Furlanetto, S., Hernquist, L., Zahn, O., Zaldarriaga, M. 2005, Ap.J., 630, 657

mcquinnMcquinn, M., Zahn, O., Zaldarraiga, M., Hernquist, L., Furlanetto, S. 2006, ApJ, in press (astroph/0512263)

Meiksin 2005Meiksin, A. 2005, MNRAS, 356: 596-606

Melchiorri et al. 2005Melchiorri, A., Choudhury, T. R., Serra, P., \& Ferrara, A. 2005, astro-ph/0506486

Mennella et al.(2004)Mennella, A., et al. 2004, AIP Conf. Proc. 703: Plasmas in the Laboratory and in the Universe: New Insights and New Challenges, 703, 401. Also, astro-ph/0310058

Mesinger, A., Haiman, Z. 2004, ApJ, 611, L69-72

Miralda-Escude 1998Miralda-Escudé, J. 1998, Ap.J., 501: 15-22

Miralda-Escudé 2003Miralda-Escudé, J. 2003, Ap.J., 597: 66-73 
Miralda-Escudé et al. 1996Miralda-Escudé, J., Cen, R., Ostriker, J. P., \& Rauch, M. 1996, Ap.J., 471: 582-616

Miralda-Escudé et al. 2000Miralda-Escudé, J., Haehnelt, M., \& Rees, M. J. 2000, Ap.J., 530: 1-16

Mobasher et al. 2005Mobasher, B. et al. 2005 ApJ, 635, 832

Montroy et al.(2005)Montroy, T., et al. 2005, astro-ph/0507514

Morales \& Hewitt 2004Morales, M. \& Hewitt, J. 2004, ApJ, 615, 7-18

Morales 2005Morales, M. 2005, ApJ, 619, 678-683

Oh 2001Oh, S. P. 2001, Ap.J., 553: 499-512

Oh 2002Oh, S. P. 2002, MNRAS, 336: 1021-1029

Oh \& Furlanetto 2005Oh, S. P., \& Furlanetto, S. R. 2005, Ap.J. Lett., 620: L9L12

Oh \& Haiman 2004Oh, S.P. \& Haiman, Z. 2004, MNRAS, 346, 456-472

Oh \& Mack 2003Oh, S.P. \& Mack, K. 2003, MNRAS, 346, 871-877

Oxley et al.(2005) Oxley, P., et al. 2005, Proc.SPIE Int.Soc.Opt.Eng. 5543 (2004) $320-331$

Piacentini et al.(2005)Piacentini F., et al. 2005, astro-ph/0507507

PierpaoliPierpaoli, E. 2003, MNRAS, 342, L63

pagePage, L., Hinshaw, G., Komantsu, E., 2006, ApJS, in press (astroph/0603450)

Panagia et al. 2005Panagia, N. et al. 2005, ApJ, 633, L1

Paschos \& Norman 2005Paschos, P., \& Norman, M. L. 2005, Ap.J., 631: 59-84

Pentericci et al. 2002Pentericci, L., et al. 2002, A.J., 123: 2151-2158

Pettini et al. 2003Pettini, M., Madau, P., Bolte, M., Prochaska, J. X., Ellison, S. L., \& Fan, X. 2003, Ap.J., 594: 695-703 
Price et al. 2005Price, P. A. et al. 2005, ApJL, submitted (astro-ph/0509697)

Rauch 1998Rauch, M. 1998, ARA\&A, 36: 267-316

Rauch et al. 1997Rauch, M., et al. 1997, Ap.J., 489: 7-20

Razoumov et al. 2002Razoumov, A. O., Norman, M. L., Abel, T., \& Scott, D. 2002, Ap.J., 572: 695-704

Readhead et al.(2004)Readhead, A. C. S., et al. 2004, Science, 306, 836

Readhead et al.(2004b)Readhead, A. C. S., et al. 2004, Ap.J., 609, 498

Rees98Rees, M.J. 1998, Proceedings of the National Academy of Sciences Colloquium: The Age of the Universe, Dark Matter, and Structure Formation, (NAS: Washington DC), p. $47-52$

Rhoads et al. 2003Rhoads, J. E., et al. 2003, A.J., 125: 1006-1013

Richards et al. 2002Richards, G. T., et al. 2002, A.J., 123: 2945-2975

Richards et al. 2005Richards, G. T., et al. 2005, AJ, submitted

Ricotti, Gnedin, \& Shull(2000)Ricotti, M., Gnedin, N. Y., \& Shull, J. M. 2000, Ap.J., 534: 41-56

Ricotti \& Ostriker 2004Ricotti, M., \& Osteriker, J. P. 2004, MNRAS, 352: 547562

Ruhl et al.(2004)Ruhl, J., et al. 2004, Proceedings of the SPIE, 5498, 11

Salvaterra et al. 2005Salvaterra, R., Haardt, F., \& Ferrara, A. 2005, MNRAS, 362, L50 - L54

Santos 2004Santos, M. R. 2004, MNRAS, 349: 1137-1152

Santos et al. 2004Santos, M. R., Ellis, R. S., Kneib, J.-P., Richard, J., \& Kuijken, K. 2004, Ap.J., 606: 683-701

Santos et al. 2005Santos, M., Cooray, A., Knox, L. 2005, ApJ, 625, 575-587

Santos et al. 2003Santos, M. G., Cooray, A. Haiman, Z., Knox, L. and Ma, C.-P., 
2003, Ap.J., 598: 756-766

Schaye et al.(2000)Schaye, J., Theuns, T., Rauch, M., Efstathiou, G., \& Sargent, W. L. W. 2000, MNRAS, 318: 817-826

Schaye et al. 2003Schaye, J., Aguirre, A., Kim, T.-S., Theuns, T., Rauch, M., \& Sargent, W. L. W. 2003, Ap.J., 596: 768-796

Scheuer 1965Scheuer, P. A. G. 1965, Nature, 207: 963-

Schiminovich et al.(2005)Schiminovich, D., et al. 2005, Ap.J. Lett., 619, L47

Schmidt et al. 1995Schmidt, M., Schneider, D. P., \& Gunn, J. E. 1995, A.J., 110: 68-77

Schneider et al. 2005Schneider, D. P., et al. 2005, A.J., 130: 367-380

Scott \& Rees 1990Scott, D., Rees, M.J. 1990, MNRAS, 247, 510-516

Seshadri \& Subramanian (1998)Seshadri, T., and Subramanian, K. 1998, Phys. Rev. D., 58, 063002

Seljak \& Zaldarriaga(1996)Seljak, U., \& Zaldarriaga, M. 1996, Ap.J., 469, 437

Seljak et al.(2005)Seljak, U., et al. 2005, Phys. Rev. D., 71, 103515

Sethi 2005Sethi, S. 2005, MNRAS, 363, 818

Shklovsky 1964Shklovsky, I. S. 1964, Astron. Zh. 41: 801

Shull et al.(2004)Shull, J. M., Tumlinson, J., Giroux, M. L., Kriss, G. A., \& Reimers, D. 2004, Ap.J., 600: 570-579

Sokasian et al. 2002Sokasian, A., Abel, T., Hernquist, L. 2002, MNRAS, 332, 601 Songaila 2004Songaila, A. 2004, A.J., 127: 2598-2603

Songaila 2005Songaila, A. 2005, A.J., in press (astro-ph/0507649)

Songaila \& Cowie 2002Songaila, A., \& Cowie, L. L. 2002, A.J., 123: 2183-2196

Songaila, Hu, Cowie, \& McMahon 1999Songaila, A., Hu, E. M., Cowie, L. L., \& McMahon, R. G. 1999, Ap.J. Lett., 525: L5-L8 
Spergel, D., Verde, L., Peiris, H. et al. ApJS, 148, 175-194

Spergel, D., Bean, R., O’Dore, M. et al. ApJS, in press (astroph/0603449

Steidel et al. (1999)Steidel, C. C., Adelberger, K. L., Giavalisco, M., Dickinson, M. and Pettini, M. 1999, Ap.J., 519, 1.

Steidel et al. 2001Steidel, C. C., Pettini, M., \& Adelberger, K. L. 2001, Ap.J., 546: $665-671$

Stern et al. 2005Stern, D., Yost, S. A., Eckart, M. E., Harrison, F. A., Helfand, D. J., Djorgovski, S. G., Malhotra, S., \& Rhoads, J. E. 2005, Ap.J., 619: 12-18

Stiavelli, Fall, \& Panagia 2004Stiavelli, M., Fall, S. M., \& Panagia, N. 2004, Ap.J. Lett., 610: L1-L4

Subrahmanyan et al. 1998Subrahmanyan, R., Kesteven, M., Ekers, R., Sinclair, M., Silk, J. 1998 MNRAS 298, 1189-1197

Subrahmanyan et al. 2005Subrahmanyan, R., Chippendale, A., Ekers, R. 2005, ATNF newsletter, 56, 18-19

Sunyaev \& Zeldovich(1980)Sunyaev, R. A., \& Zeldovich, I. B. 1980, MNRAS, 190,413

Tagliaferri et al. 2005Tagliaferri, G. et al. 2005, A\&A, 443, L1

Taniguchi et al. 2005Taniguchi, Y., et al. 2005, PASJ, 57: 165-182

Tegmark et al.(2004)Tegmark, M., et al. 2004, Phys. Rev. D., 69, 103501

Theuns et al. 2002aTheuns, T., Schaye, J., Zaroubi, S., Kim, T.-S., Tzanavaris, P., \& Carswell, B. 2002,

Theuns et al.(2002b)Theuns, T., Bernardi, M., Frieman, J., Hewett, P., Schaye, J., Sheth, R. K., \& Subbarao, M. 2002, Ap.J. Lett., 574: L111-L114

Ap.J. Lett., 567: L103-L106

Thompson et al. 2005Thompson, R. I., et al. 2005, A.J., 130: 1-12 
Totani et al. 2006Totani, T., et al. 2006, PASJ, in press

Tozzi et al. 1999Tozzi, P., Madau, P., Meiksin, A., Rees, M. 2000, ApJ, 528, $597-606$

Tran et al. 2004Tran, K.-V. H., Lilly, S. J., Crampton, D., \& Brodwin, M. 2004, Ap.J. Lett., 612: L89-L92

Venkatesan et al. 2001Venkatesan, A., Giroux, M. L., \& Shull, J. M. 2001, Ap.J., 563: $1-8$

Walter et al. 2003Walter, F., Bertoldi, F., Carilli, C., et al. 2003, Nature, 424, 406-408

Walter et al. 2004Walter, F., Carilli, C., Bertoldi, F. et al. 2004, ApJ, 615, L17-20

Wang et al. 2005Wang, X., Tegmark, M., Santos, M., Knox, L. 2005, ApJ, in press (astroph-0501081)

Wang \& Hu 2005Wang, X., Hu, W. 2005, ApJ, in press (astroph/0511141)

Warren et al. 1994Warren, S. J., Hewett, P. C., \& Osmer, P. S. 1994, Ap.J., 421: $412-433$

Webb et al. 1992Webb, J. K., Barcons, X., Carswell, R. F., \& Parnell, H. C. 1992, MNRAS, 255: 319-324

Weinberg et al. 1997Weinberg, D. H., Miralda-Escude, J., Hernquist, L., \& Katz, N. 1997, Ap.J., 490: 564-570

Weiss et al.(2005)Weiss, R., et al. 2003, http://www.nsf.gov/mps/ast/tfcr.jsp

White et al. 2003White, R. L., Becker, R. H., Fan, X., \& Strauss, M. A. 2003, A.J., 126: 1-14

White et al. 2005White, R. L., Becker, R. H., Fan, X., \& Strauss, M. A. 2005, A.J., 129: 2102-2107

Willott et al. 2005Willott, C. J., Percival, W. J., McLure, R. J., Crampton, D., 
Hutchings, J. B., Jarvis, M. J., Sawicki, M., \& Simard, L. 2005, Ap.J., 626: $657-665$

Wolf et al. 2003Wolf, C., Wisotzki, L., Borch, A., Dye, S., Kleinheinrich, M., \& Meisenheimer, K. 2003, A\&A, 408: 499-514

Wouthuysen 1952Wouthuysen, S. 1952, AJ, 57, 31-33

Wyithe \& Loeb 2003Wyithe, J. S. B., \& Loeb, A. 2003, ApJ, 586, 693

Wyithe \& Loeb 2004Wyithe, J. S. B., \& Loeb, A. 2004, Nature, 432: 194-196

Wyithe \& Loeb 2005aWyithe, J. S. B., \& Loeb, A. 2005a, Ap.J., 625: 1-5

Wyithe \& Loeb 2005bWyithe, J. S. B., \& Loeb, A. 2005b, ApJ, 625, 1

Wyithe et al. 2005Wyithe, J.S., Loeb, A., Carilli, C. 2005, ApJ, 628, 575-582

Wyithe \& Loeb 2004Wyithe, J.S., Loeb, A. 2004b, ApJ, 610, 117

Wyithe et al. 2005bWyithe, J.S., Loeb, A., Barnes, D. 2005, ApJ, 634, 715

Yan \& Windhorst 2004aYan, H., \& Windhorst, R. A. 2004a, Ap.J. Lett., 600: L1-L5

Yan \& Windhorst 2004bYan, H., \& Windhorst, R. A. 2004b, Ap.J. Lett., 612: L93-L96

York et al. 2000York, D. G., et al. 2000, A.J., 120: 1579-1587

Yu \& Lu 2005Yu, Q., \& Lu, Y. 2005, Ap.J., 620, 31

Zahn et al.(2005)Zahn, O., Zaldarriaga, M., Hernquist, L., \& McQuinn, M. 2005, Ap.J., 630, 657

Zaldarriaga \& Seljak(1997)Zaldarriaga, M., \& Seljak, U. 1997, Phys. Rev. D., 55, 1830

Zaldarriaga(1997)Zaldarriaga, M. 1997, Phys. Rev. D., 55, 1822

Zaldarriaga \& Seljak(1998)Zaldarriaga, M., \& Seljak, U. 1998, Phys. Rev. D., 58, 023003 
Zaldariagga et al. 2004aZaldarigga, M., Furlanetto, S., Henquist, L. 2004, ApJ, $608,622-635$

Zaldariagga et al. 2004bZaldarigga, M., Furlanetto, S., Loeb, A. 2004, ??

Zhang, Anninos, \& Norman 1995Zhang, Y., Anninos, P., \& Norman, M. L. 1995, Ap.J. Lett., 453: L57- L60

Zhang et al.(2004)Zhang, P., Pen, U., Trac, H. 2004, MNRAS, 355, 451 - 460

Zheng et al.(2004)Zheng, W., et al. 2004, Ap.J., 605: 631-644

Zygelman 2005Zygelman, B. 2005, ApJ, 622, 1356-1362 


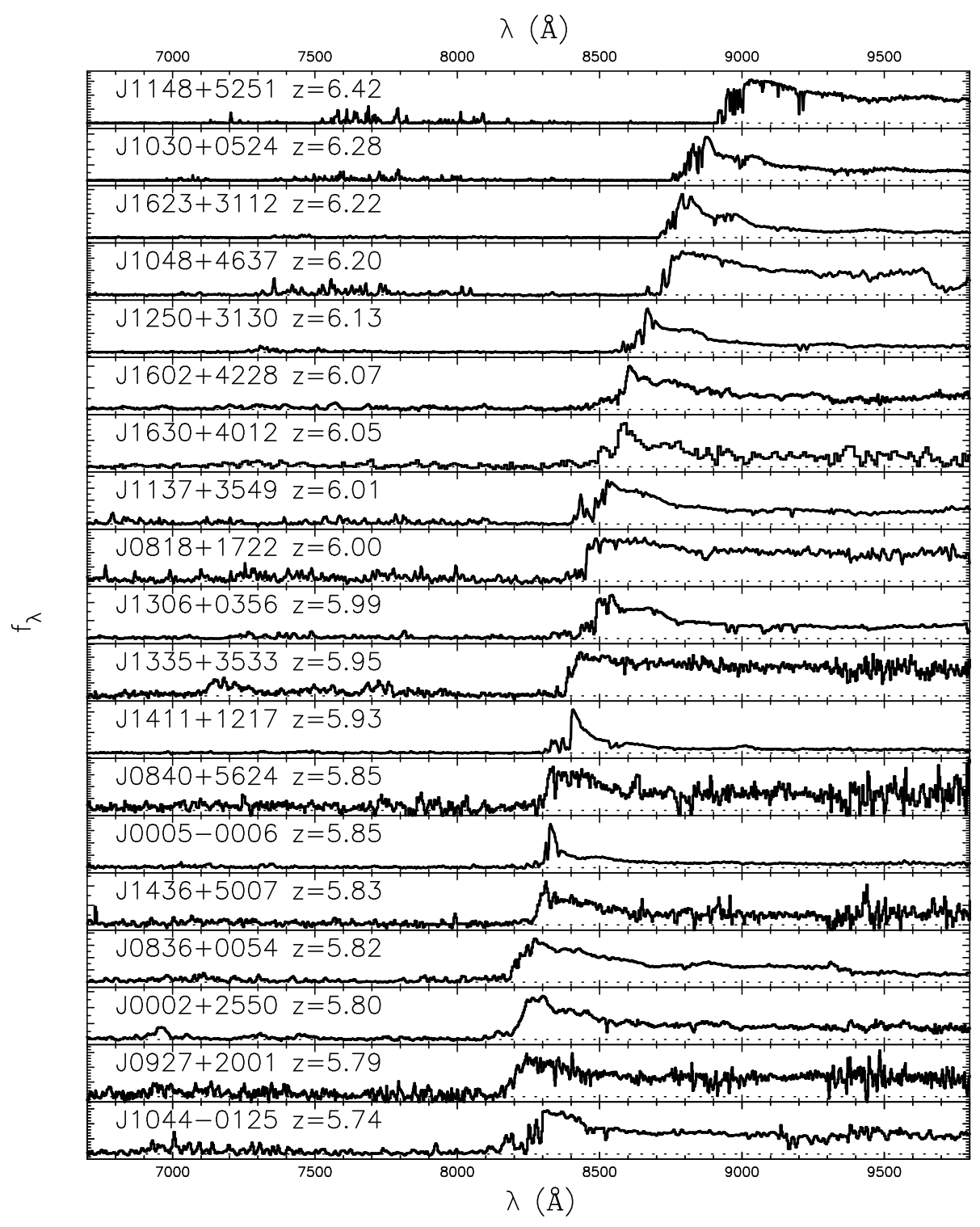

Figure 1: Moderate resolution spectra of nineteen SDSS quasars at $5.74<z<$ 6.42. Adapted from Fan et al. (2006b) 


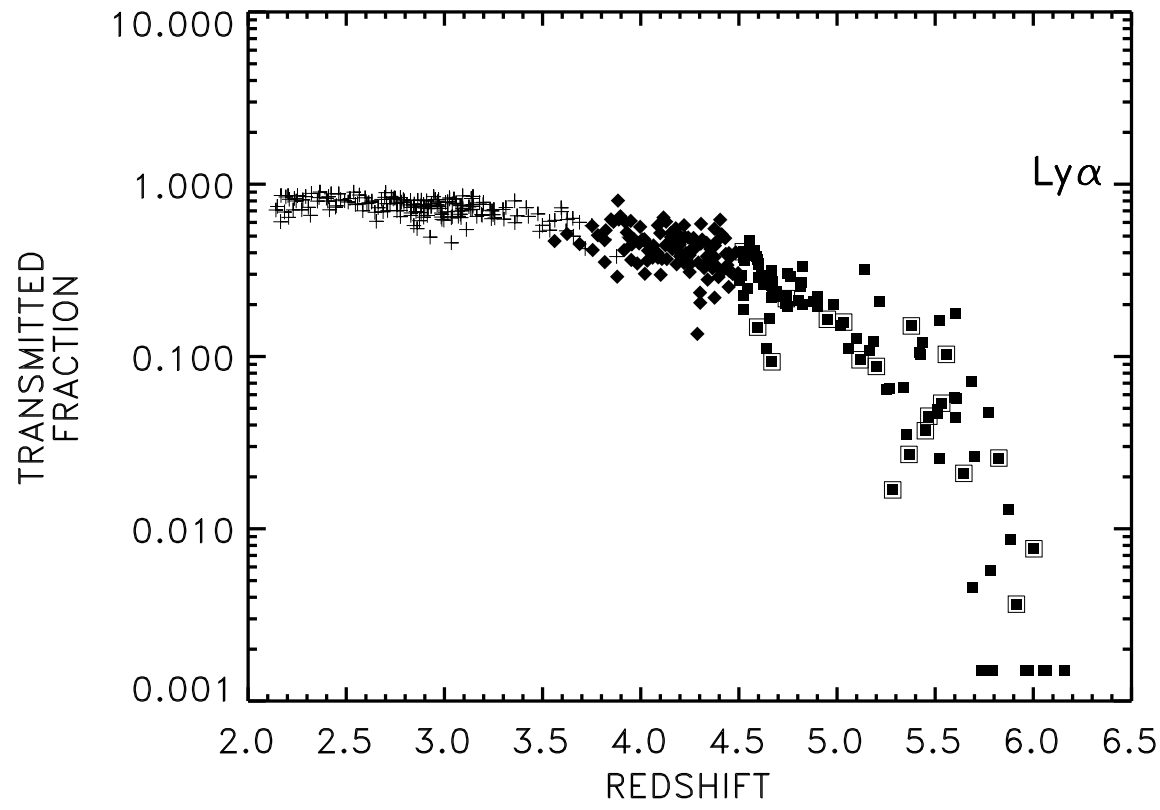

Figure 2: Transmitted flux blueward of Ly $\alpha$ emission as a function of redshift from $z \sim 2$ to 6.3 using Keck/ESI and HIRES data. Large open squares are points data for possible BAL quasars. Flux was computed in bins of $15 \AA$. Adapted from Songaila (2004) 


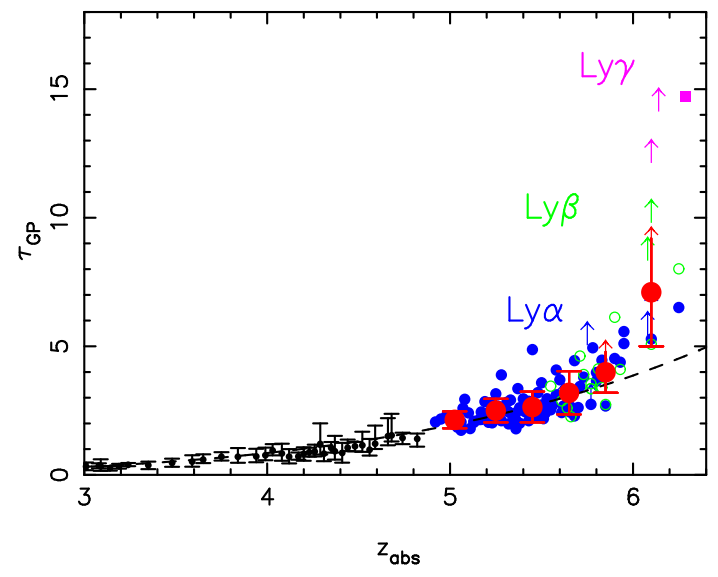

Figure 3: Evolution of Optical depth with combined Ly $\alpha$ and $\operatorname{Ly} \beta$ results. The dash line is for a redshift evolution of $\tau_{\mathrm{GP}} \propto(1+z)^{4.3}$. At $z>5.5$, the best fit evolution has $\tau_{\mathrm{GP}} \propto(1+z)^{>10.9}$, indicating an accelerated evolution. The large open symbols with error bars are the average and standard deviation of optical depth at each redshift. The sample variance increases also increases rapidly with redshift. Adapted from Fan et al. (2006b). 


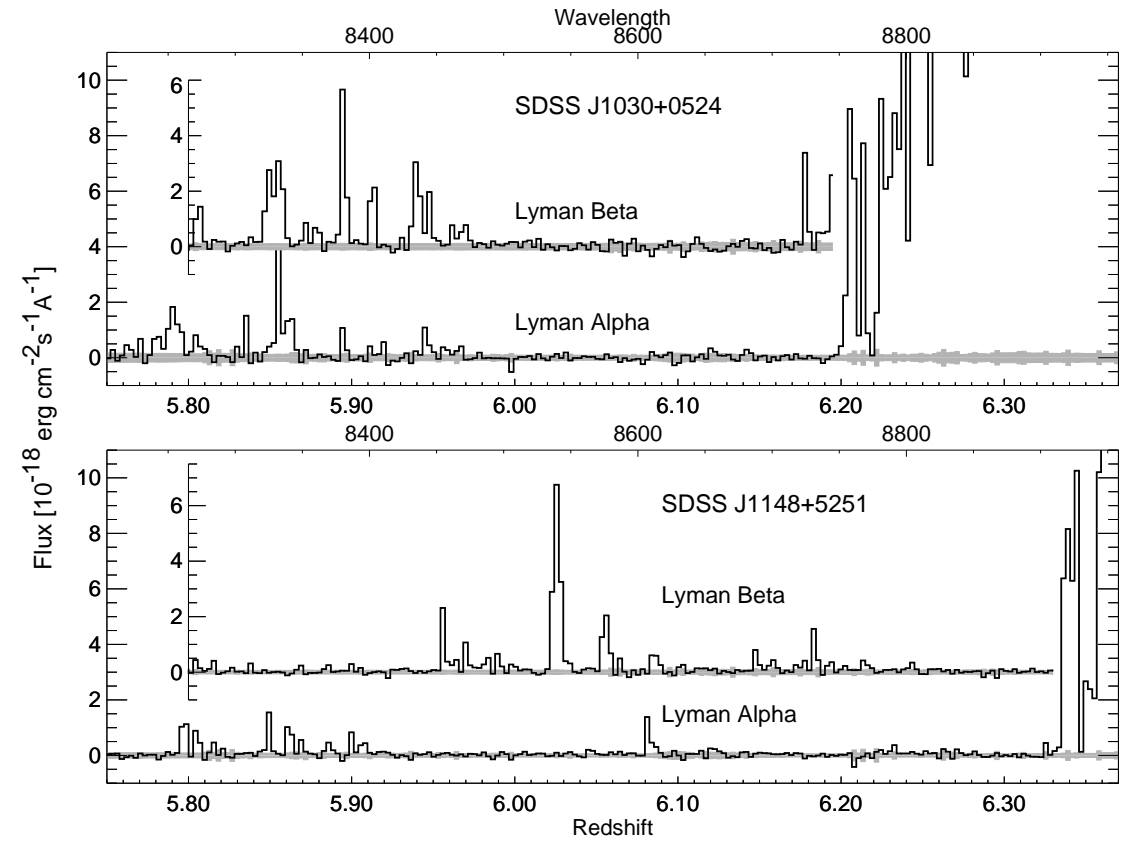

Figure 4: Close-up of the $\operatorname{Ly} \alpha$ and $\operatorname{Ly} \beta$ troughs in the two highest redshift quasars currently known. SDSS J1030+0524 $(z=6.28)$ shows complete Gunn-Peterson absorption in both Ly $\alpha$ and $\operatorname{Ly} \beta$, where SDSS J1148+5251 $(z=6.42)$ has clear transmission, suggesting large line of sight variance at the end of reionization. Adapted from White et al. (2003). 


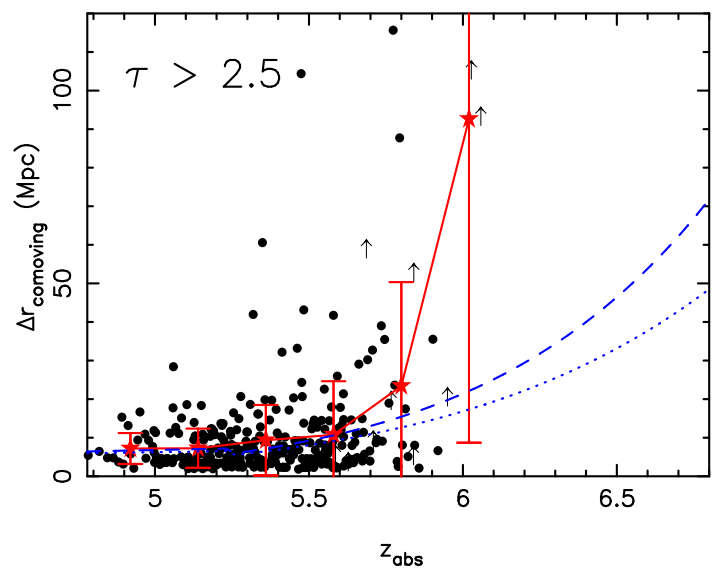

Figure 5: Distributions of dark gaps, defined as regions in the spectra where all pixel having observed optical depth larger than 2.5 for Ly $\alpha$ transition. Upward arrows are gaps immediately blueward of quasar proximity zone, therefore the length is only a lower limit. Solid lines with error bars are average depth lengths with 1- $\sigma$ dispersion at each redshift bin. Long dark gaps start to appear at $z \sim 5.6$, with the average gap length increases rapidly at $z>6$, marking the end of reionization. It is compared with simulation of Paschos \& Norman (2005), in which dashed and dotted lines are for moderate and high spectral resolutions. The simulation has an overlapping redshift at $z \sim 7$. Adapted from Fan et al. (2006b). 


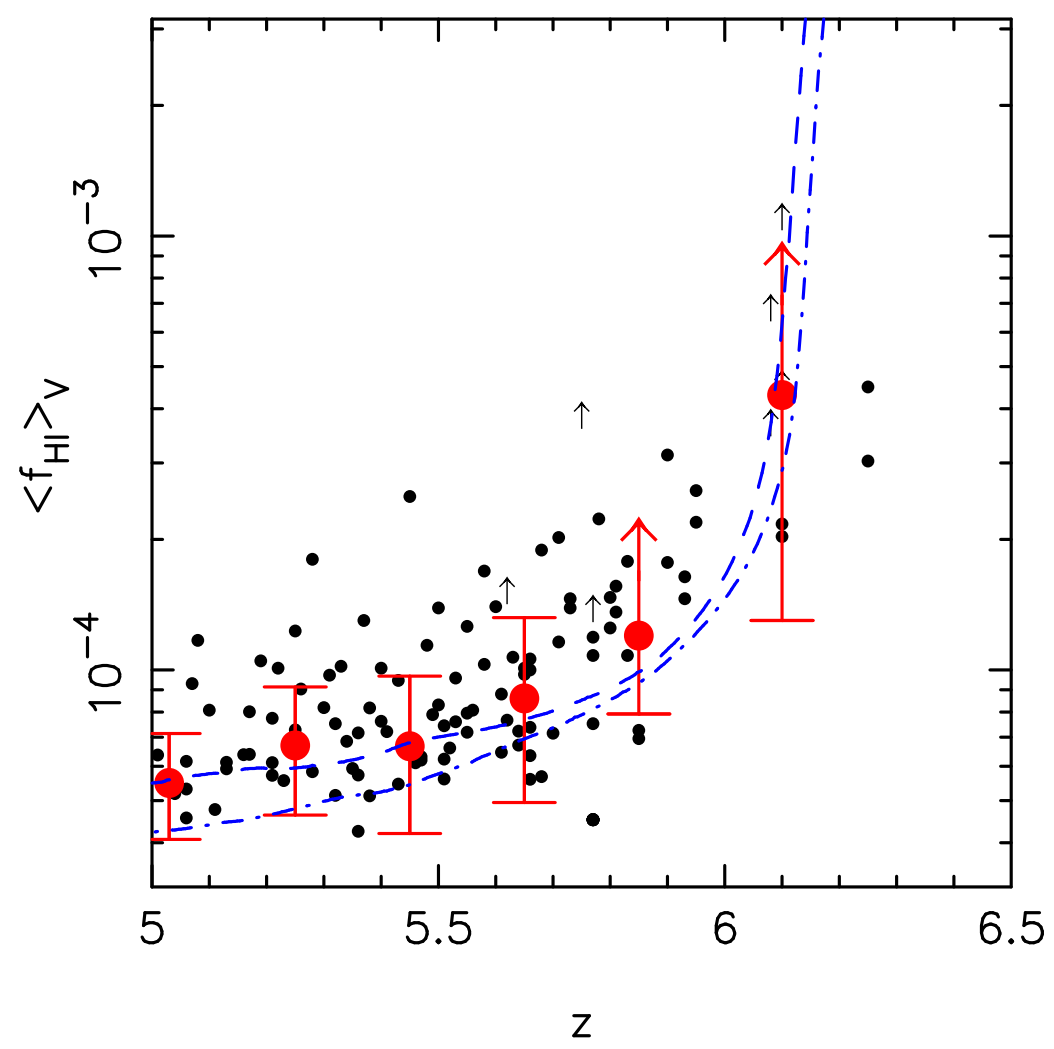

Figure 6: Evolution of volume-averaged neutral hydrogen fraction of the IGM. The solid points with error bars are measurements based on the nineteen highredshift quasars. The dashed and dashed-dotted-dashed lines is volume-averaged results from the A4 and A8 simulations of Gnedin (2004). The neutral fraction inferred from the observations is comparable to that of the transition from overlapping stage to post-overlap stage of reionization in simulations (Gnedin 2000). Adapted from Fan et al. (2006b) 

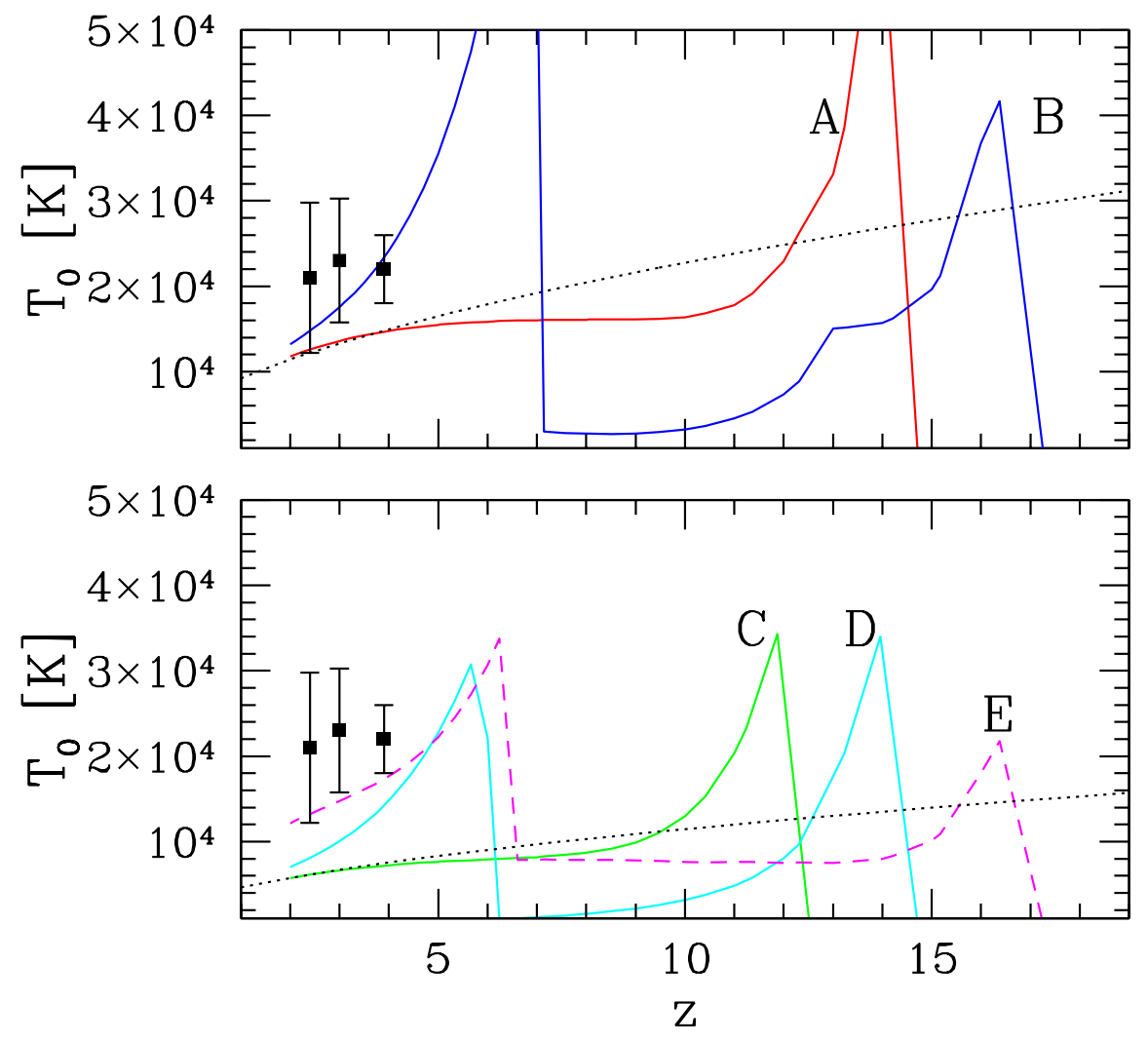

Figure 7: Evolution of IGM temperature for a quasar-like ionizing spectrum, assuming different reionization history. In models where the Universe is reionized early and remained such afterwards, the IGM temperature is too cold comparing to measurements at $z=2-4$. Model $\mathrm{A}$ has a single-episode reionization at $z=14$ and remains highly ionized thereafter. Model B also has early reionization, but experienced a drop in ionizing flux until $z \sim 7$ at which it undergoes a second period of reionization. To prevent overcooling, a major heating event that resulted in large change in the IGM ionization state would have to occur at $z=6-10$. However, different history of HeII reionization history may complicate this intepretation. Adapted from Hui \& Haiman (2003). 


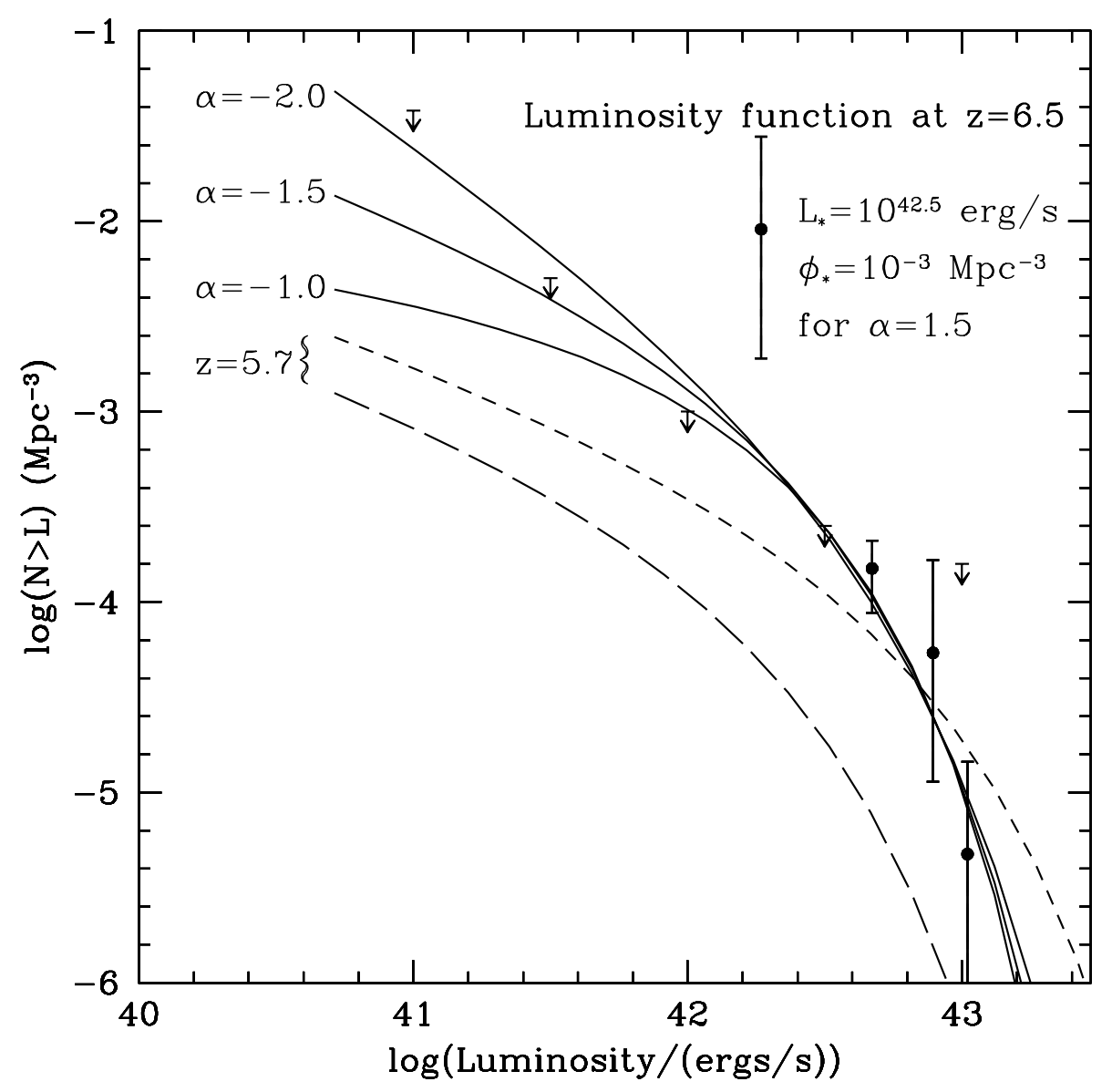

Figure 8: The luminosity function of Ly $\alpha$ galaxies at $z \sim 6.5$ and $z \sim 5.7$, from the complications in Malhotra \& Rhoads (2004). The luminosity function shows little evolution between these redshifts, consistent with a largely ionized IGM at $z \sim 6.5$. Adapted from Malhotra \& Rhoads (2004). 


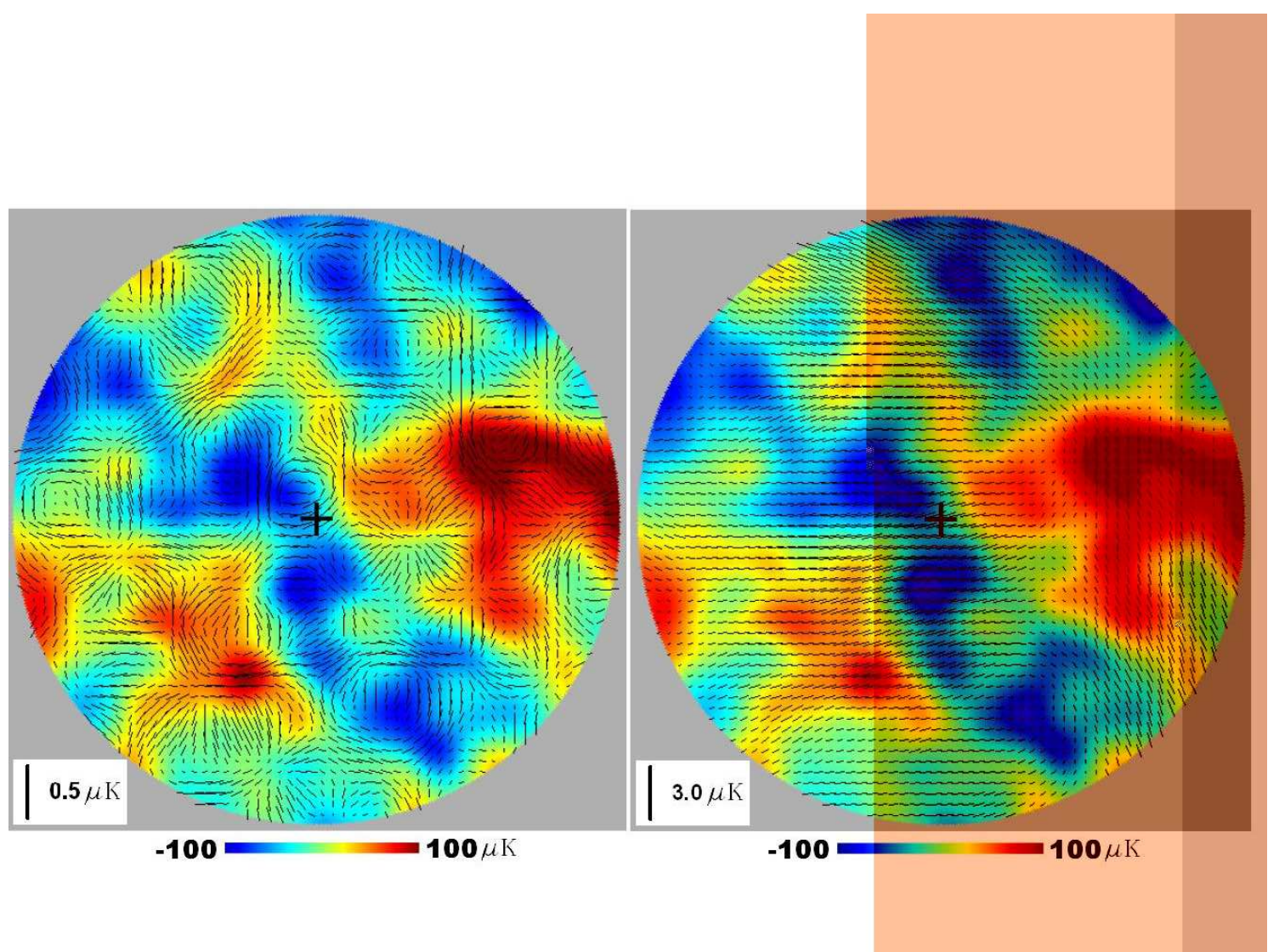

Figure 9: Large angular scale temperature (color scale) and polarization (lines) simulations in real-space. The left panel shows a simulation with no reionization and the right panel shows the effects of instantaneous reionization with $\tau=0.17$. Both maps and CMB observables are smoothed with a $4^{\circ}$ beam. Reionization does not affect the large-scale temperature pattern but it produces roughly tentimes larger polarization at large scales - note the change in scale for polarization between the two panels. The maximum polarization in the right panel is $\simeq 800$ nK. Figures courtesy of Eric Hivon. 


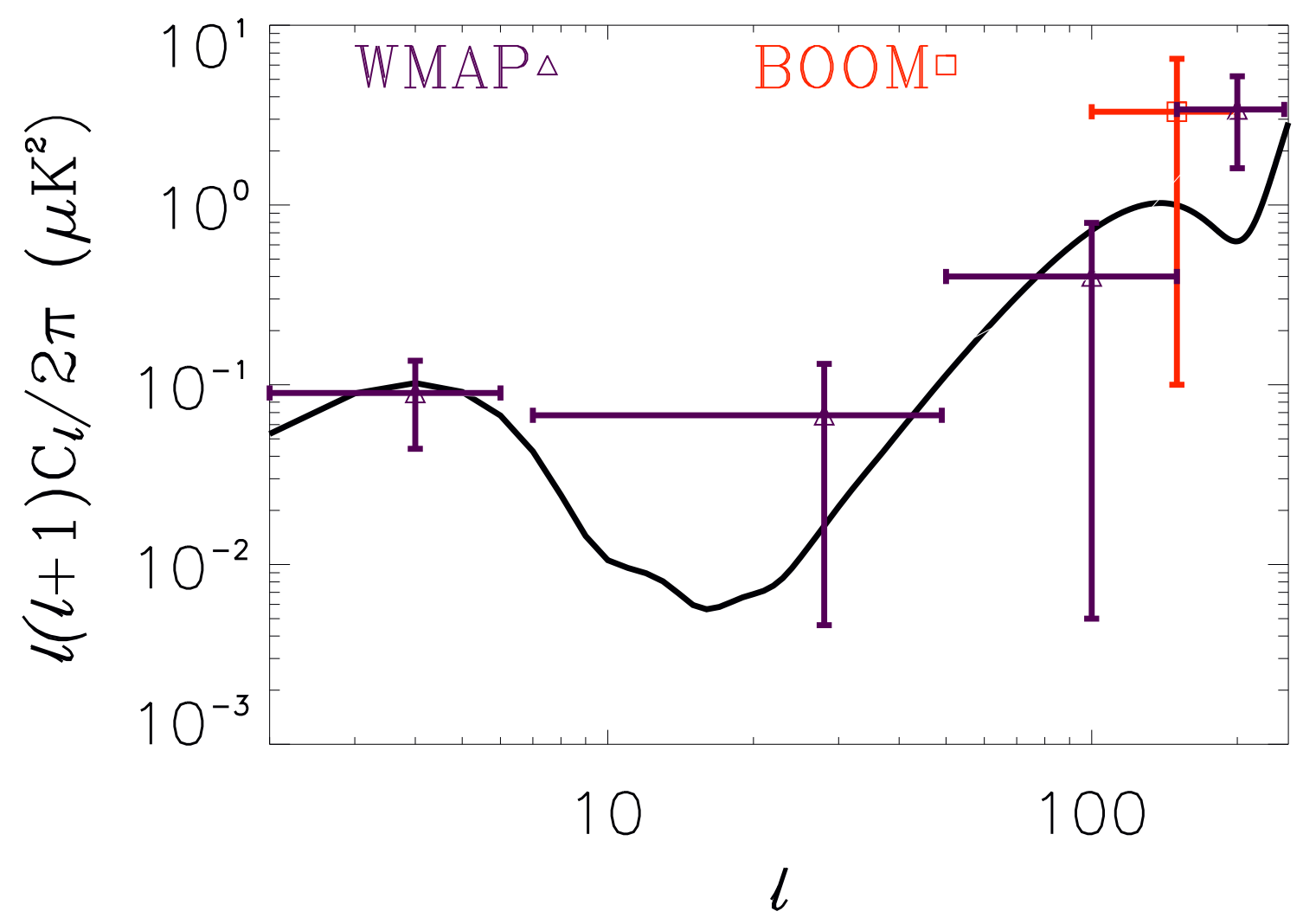

Figure 10: Measurements of the large-angular scale gradient-mode polarization power spectrum $C_{\ell}^{E}$ for angular scales greater than $1^{\circ} \Leftrightarrow \ell \lesssim 200$. The solid line is the polarization power spectrum for the WMAP best fit cosmological model, with $\tau=0.09$ (4, page) The dashed line displays the polarization power spectrum for a model generated using the WMAP cosmological parameters but with $\tau=0$, which is consistent with the E-mode detections by BOOMERANG, CBI, DASI, and CAPMAP and the upper limit of POLAR. 


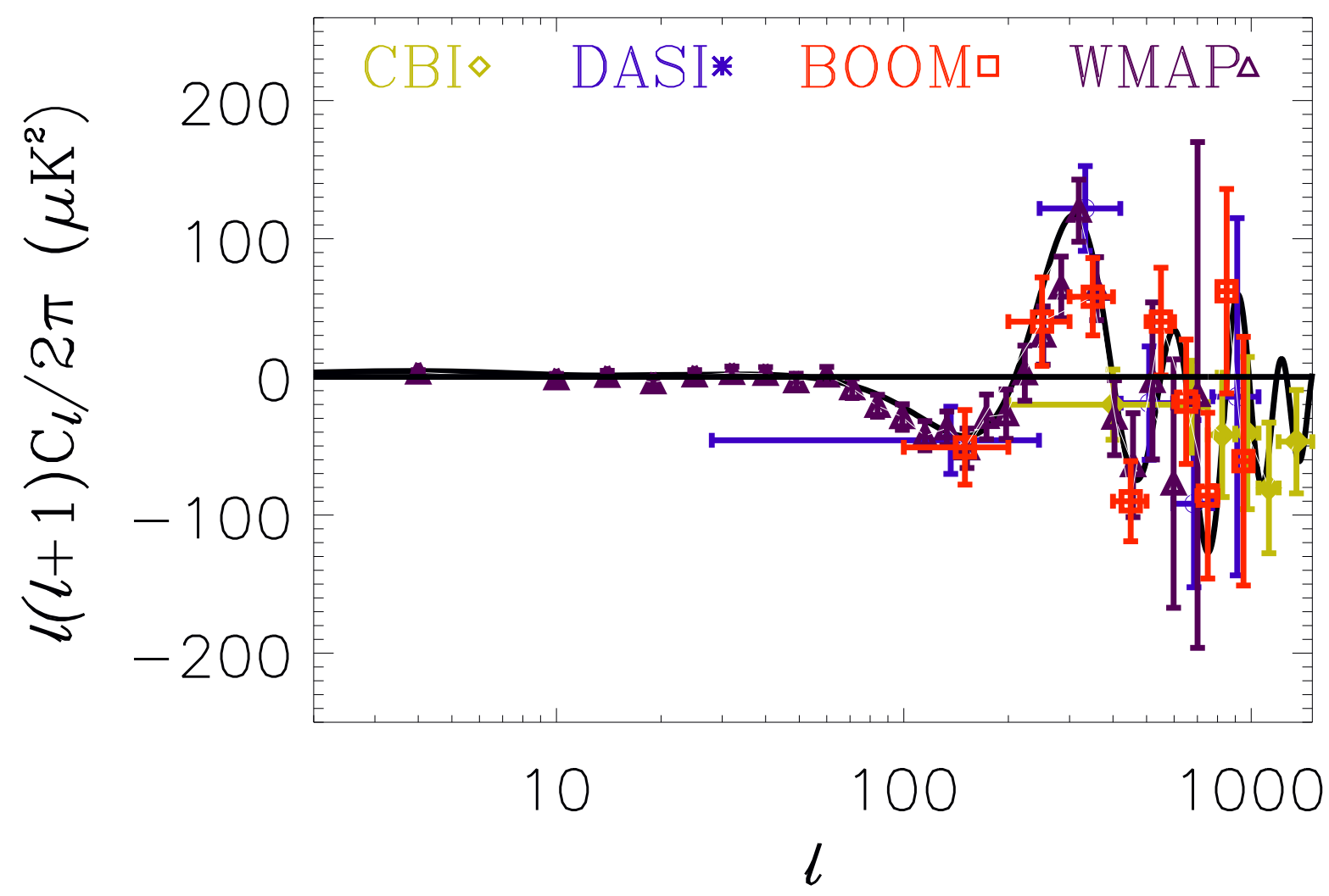

Figure 11: Measurements of the temperature-polarization cross-correlation power spectrum $C_{\ell}^{T E}$. The solid line is the power spectrum for the WMAP best fit cosmological model, with $\tau=0.09$ (4, page). 


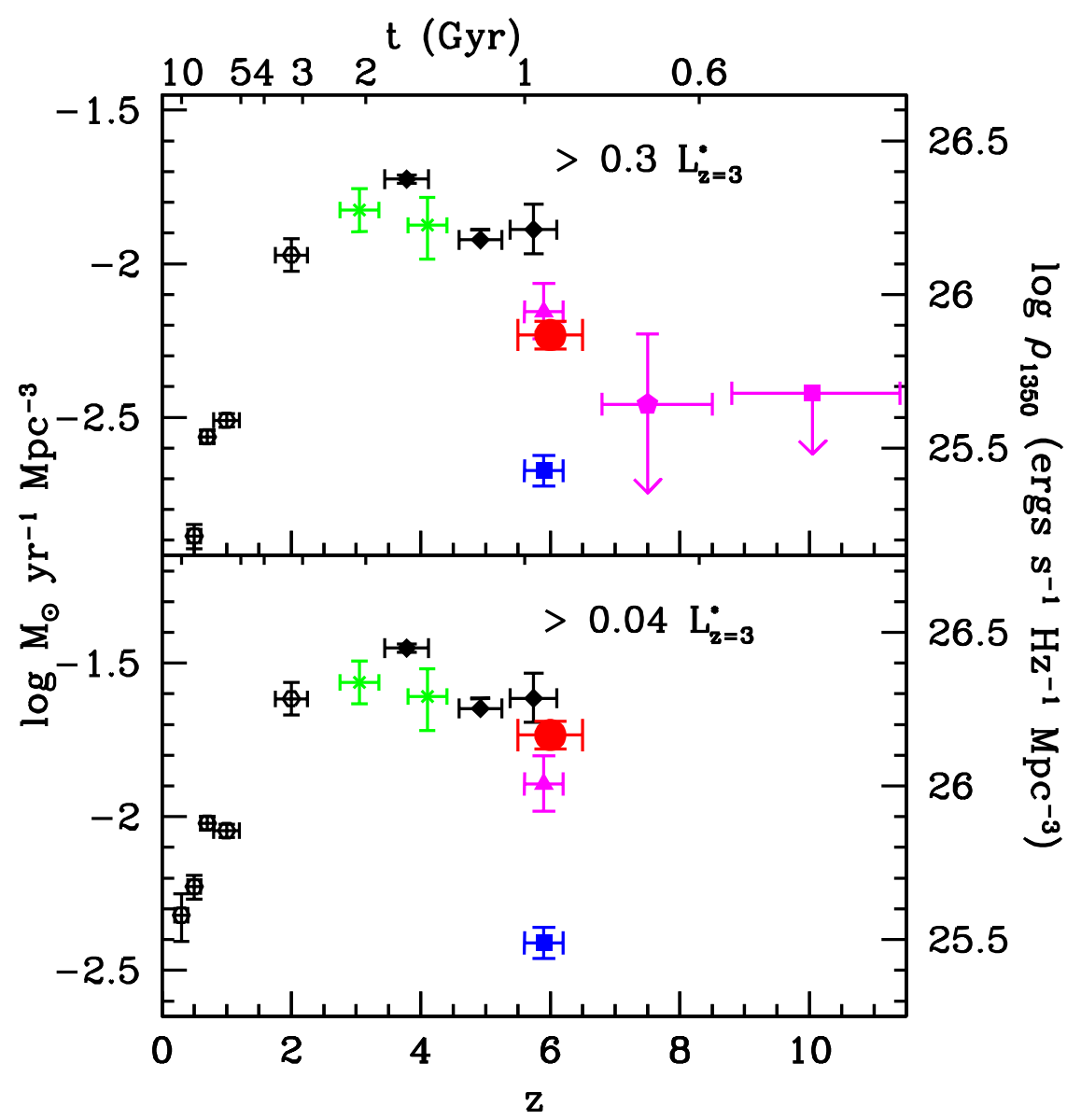

Figure 12: The cosmic star formation history (uncorrected for extinction) integrated down to $0.3 L_{z=3}^{*}$ (top panel) and $0.04 L_{z=3}^{*}$ (bottom panel). These luminosities correspond to the faint-end limits for $z_{850}$ and $i_{775}$-dropout probes at $z \sim 7-8$ and $z \sim 6$, respectively. The large red circle denotes determination at $z \sim 6$ from Bouwens et al. (2006). and compared with previous determinations by Schiminovich et al. (2005) (open squares), Steidel et al. (1999) (green crosses), Giavalisco et al. (2004b) (black diamonds), Bouwens et al. 2004a (magenta triangle), Bunker et al. (2004) (blue square), Bouwens et al. (2004b) (magenta pentagon), and Bouwens et al. (2005) (magenta square). The figure is divided into two panels to illustrate how much stronger the evolution is at the bright end of the LF ( $\left.\gtrsim 0.3 L_{z=3}^{*}\right)$ than it is when integrated to the $i$-dropout faint-end limit $\left(0.04 L_{z=3}^{*}\right)$. Adapted from Bouwens et al. (2005). 

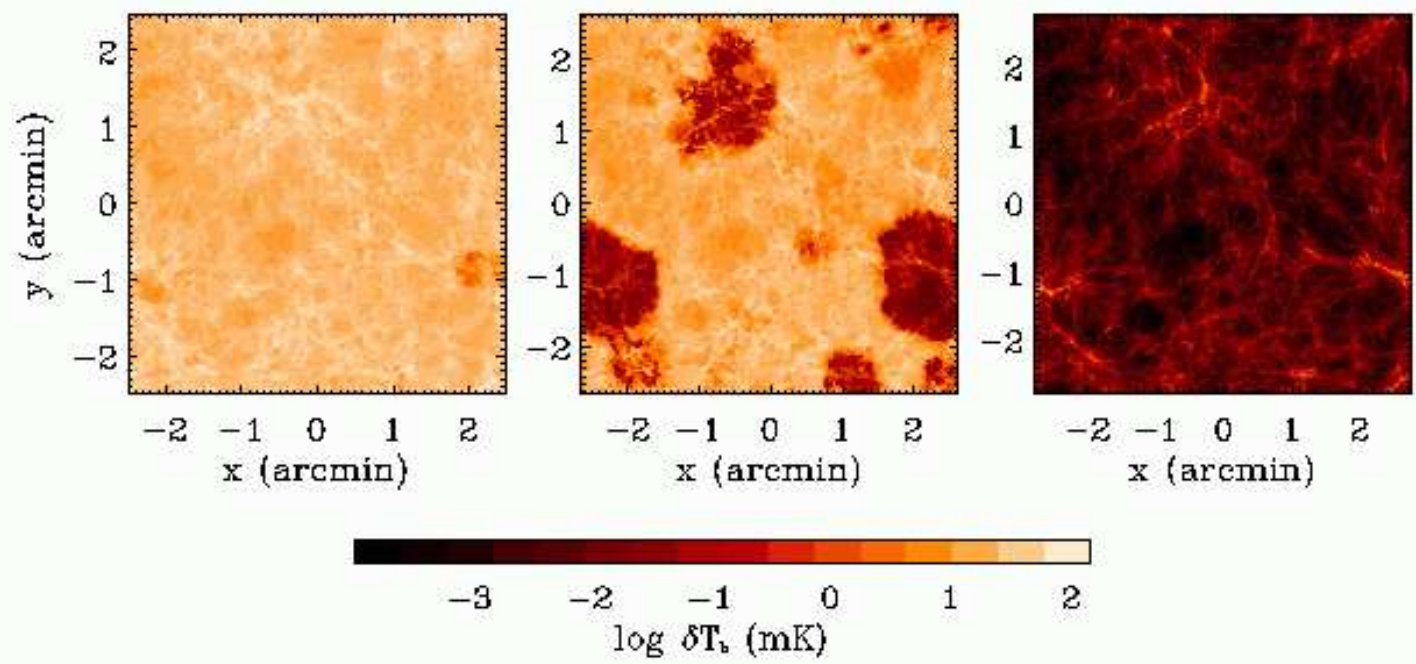

Figure 13: The simulated HI $21 \mathrm{~cm}$ brightness temperature distribution during reionization at $z=12,9,7$ (Furlanetto et al. 2004a; Zaldariagga et al. 2004a). 

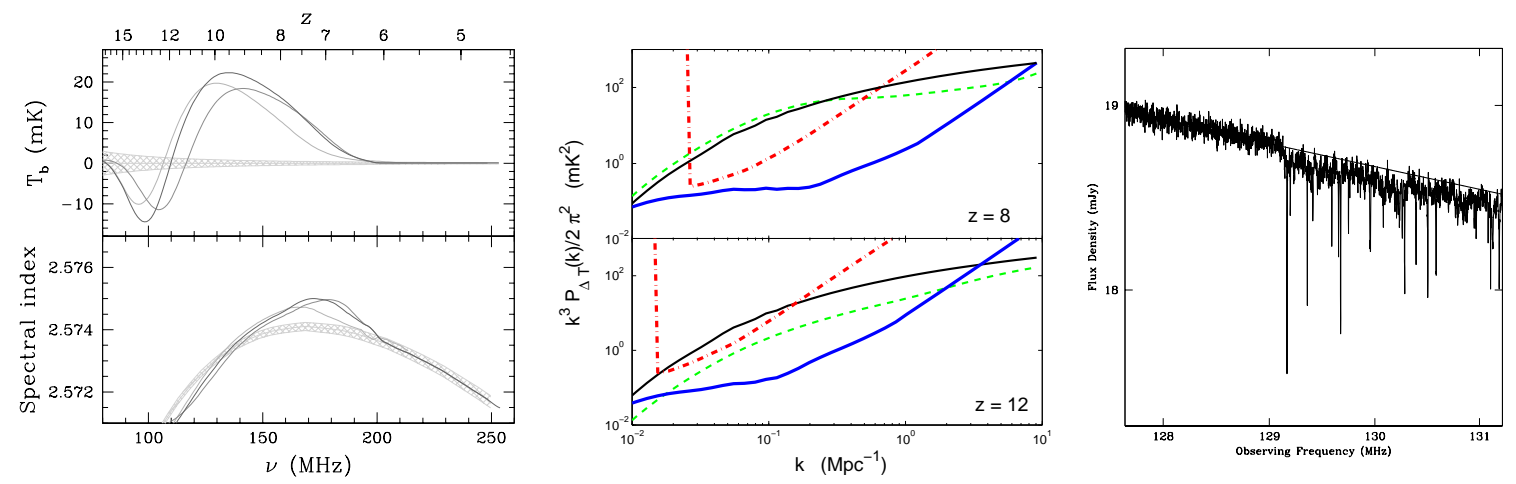

Figure 14: Left: Global (all sky) HI signal from reionization (Gnedin \& Shaver 2003). The shaded region shows the expected thermal noise in a carefully controlled experiment. Center: Predicted HI $21 \mathrm{~cm}$ brightness temperature power spectrum (in $\log$ bins) at redshifts 8 and 12 (McQuinn et al. 2006). The thin black line shows the signal when density fluctuations dominate. The dashed green line shows the predicted signal for $\bar{x}_{i}=0.2$ at $z=12$, and $\bar{x}_{i}=0.6$ at $z=8$, in the Furlanetto et al. (2004) semi-analytic model. The thick blue line shows the SKA sensitivity in $1000 \mathrm{hrs}$. The thick red dot-dash show the sensitivity of the pathfinder experiment LOFAR. The cutoff at low $\mathrm{k}$ is set by the primary beam. Right: The simulated SKA spectrum of a radio continuum source at $z=10$ (Carilli et al. 2002). The straight line is the intrinsic power law (synchrotron) spectrum of the source. The noise curve shows the effect of the $21 \mathrm{~cm}$ line in the neutral IGM, including noise expected for the SKA in a 100 hour integration. 


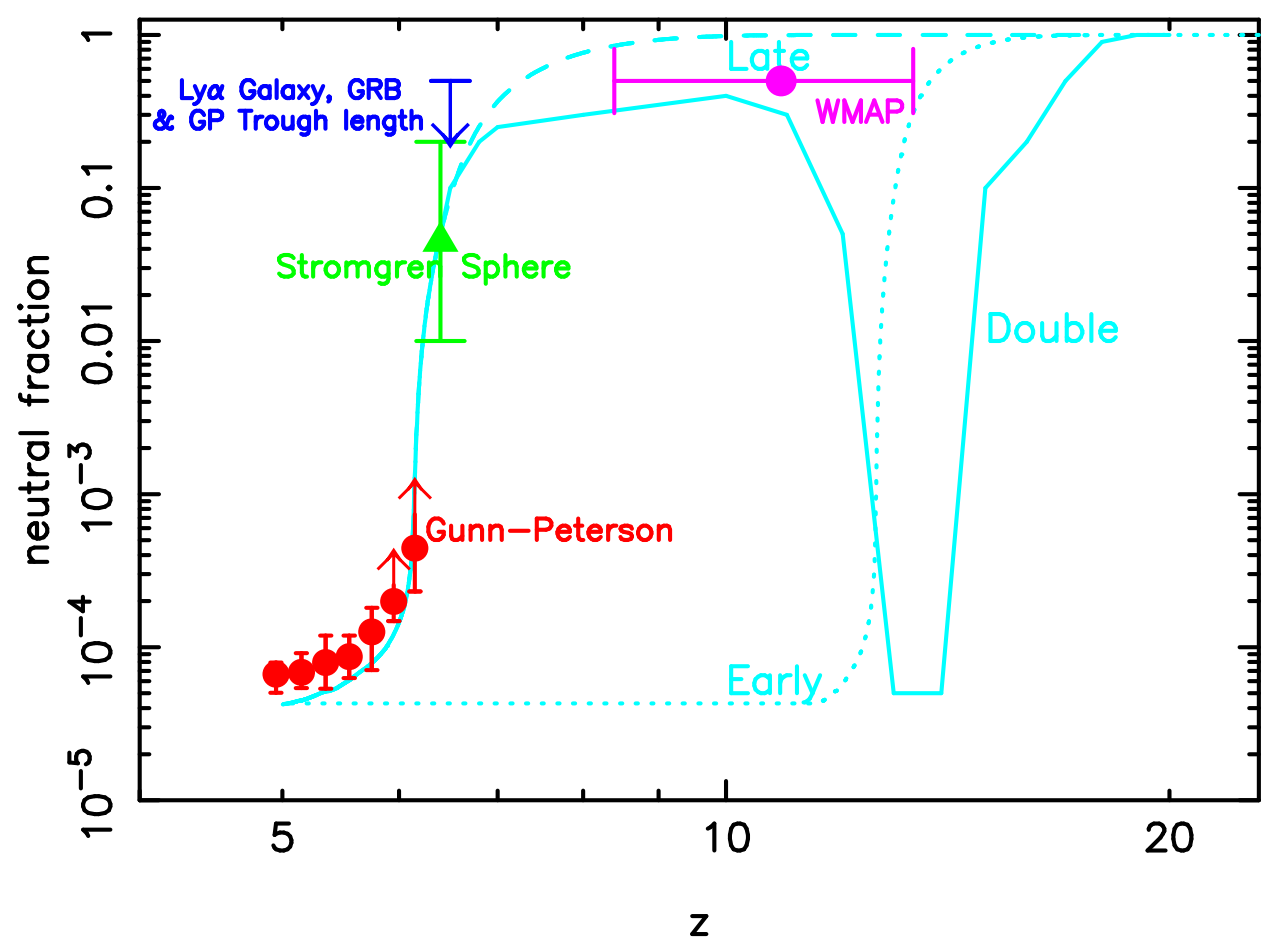

Figure 15: The volume averaged neutral fraction of the IGM versus redshift using various techniques. The dash line shows the fiducial model of Gnedin (2004) with late reionization at $z=6-7$, the solid line shows an idealized model with double reionization as described in Cen (2003a), and the dotted line illustrates the model with early reionization at $z \sim 14$. 